\title{
miR-142 controls metabolic reprogramming that regulates dendritic cell activation
}

\author{
Yaping Sun, ${ }^{1}$ Katherine Oravecz-Wilson, ${ }^{1}$ Sydney Bridges, ${ }^{2}$ Richard McEachin, ${ }^{3}$ Julia Wu, ${ }^{1}$ Stephanie H. Kim, ${ }^{1}$ Austin Taylor, ${ }^{1}$ \\ Cynthia Zajac, ${ }^{1}$ Hideaki Fujiwara, ${ }^{1}$ Daniel Christopher Peltier, ${ }^{4}$ Thomas Saunders, ${ }^{5}$ and Pavan Reddy ${ }^{1,4}$
}

'Department of Internal Medicine, Division of Hematology/Oncology, University of Michigan, Ann Arbor, Michigan, USA. ${ }^{2}$ Michigan Metabolomics and Obesity Center, ${ }^{3}$ Department of Computational Medicine and Bioinformatics, ${ }^{4}$ Department of Pediatrics, and ${ }^{5}$ Transgenic Animal Model Core, University of Michigan Medical School, Ann Arbor, Michigan, USA.

\begin{abstract}
DCs undergo metabolic reprogramming from a predominantly oxidative phosphorylation (OXPHOS) to glycolysis to mount an immunogenic response. The mechanism underpinning the metabolic reprogramming remains elusive. We demonstrate that miRNA-142 (miR-142) is pivotal for this shift in metabolism, which regulates the tolerogenic and immunogenic responses of DCs. In the absence of miR-142, DCs fail to switch from OXPHOS and show reduced production of proinflammatory cytokines and the ability to activate $T$ cells in vitro and in in vivo models of sepsis and alloimmunity. Mechanistic studies demonstrate that miR-142 regulates fatty acid (FA) oxidation, which causes the failure to switch to glycolysis. Loss- and gain-of-function experiments identified carnitine palmitoyltransferase -1a (CPT1a), a key regulator of the FA pathway, as a direct target of miR142 that is pivotal for the metabolic switch. Thus, our findings show that miR-142 is central to the metabolic reprogramming that specifically favors glycolysis and immunogenic response by DCs.
\end{abstract}

\section{Introduction}

DCs regulate both immunogenic and tolerogenic responses $(1,2)$. Metabolic processes are key to determining the functional fate of DCs upon activation (3-7). A shift toward glycolysis and the use of glucose as the preferred carbon source promotes an immunogenic or proinflammatory state in DCs $(3,4,8,9)$. On the other hand, oxidative phosphorylation (OXPHOS) and fatty acid oxidation (FAO) favor tolerogenic DCs $(3,4,7,10)$. This dichotomous metabolic reprogramming drives differentiation, and functions of DCs are crucial for the regulation of immunity $(3,4,7,10)$. Yet the mechanistic basis for this intriguing metabolic shift remains obscure.

miRs play critical roles in many cellular processes, including immunity (11-13). Earlier studies showed that miRNA-142 (miR142), a hematopoietic miR (14) is critical for governing $\mathrm{T}$ cell functions (15-17), macrophage differentiation (18), IL-6 expression in DCs (14-16), and CD4+ DC homeostasis (19). miRs have been shown to alter metabolic processes that are central to various disease processes (20-27). However, it remains unknown whether miR-mediated regulation of immune responses is linked to their ability to regulate metabolic reprogramming.

Here, we demonstrate that miR-142 plays a critical role in reprogramming DC metabolism after its activation. Absence of miR-142 caused persistence of OXPHOS and an immature tolerogenic state, with a reduction in secretion of proinflammatory cytokines, an increase in endotoxin-induced mortality in vivo, and attenuation of activation of allogeneic $\mathrm{T}$ cells in vitro and in vivo.

Conflict of interest: The authors have declared that no conflict of interest exists. Copyright: (5) 2019, American Society for Clinical Investigation.

Submitted: July 26, 2018; Accepted: February 26, 2019; Published: April 8, 2019.

Reference information: J Clin Invest. 2019;129(5):2029-2042.

https://doi.org/10.1172/JCl123839.
The specificity of miR-142 in controlling this metabolic switch and functions was demonstrated by rescuing its expression in DCs. Further mechanistic studies demonstrate that miR-142 deficiency in DCs augments OXPHOS by promoting increased FAO by direct targeting of key enzymes in the fatty acid (FA) pathway.

\section{Results}

miR-142 modulates DC responses in vitro and in vivo. We first determined whether miR-142 has a role in the DC responses by comparing the baseline phenotype, numbers, and function of DCs in the absence of miR-142. The numbers of CD11 $\mathrm{c}^{+}$DCs (Figure 1A) and the $\mathrm{CD}^{+}$and $\mathrm{CD} 8^{+} \mathrm{CD} 11 \mathrm{c}^{+} \mathrm{DC}$ subsets were similar in the $m i R$ $142^{-/-} \mathrm{C} 57 \mathrm{BL} / 6$ (B6) and WT B6 mice (Supplemental Figure 1; supplemental material available online with this article; https://doi. org/10.1172/JCI123839DS1) (19). We next determined the in vitro function of $\mathrm{CD}_{11 \mathrm{c}^{+}}$DCs harvested from WT and $m i R-142^{-\gamma_{-}} \mathrm{B} 6$ mice after TLR4 stimulation with LPS. Compared with WT DCs, the $m i R-142^{-/-}$DCs expressed significantly lower levels of MHC class II and CD80 and CD40 at steady state and after LPS stimulation (Figure 1B) with no differences in apoptosis (Figure 1B). They produced lower levels of proinflammatory TNF- $\alpha$, but higher levels of immunoregulatory cytokines IL-10 and TGF- $\beta$ and, also consistent with previous reports, higher levels of IL-6 than WT DCs (Figure 1C). Next, to determine whether the lack of miR142-altered DC responses was not limited to TLR4 stimulation by LPS, we activated the DCs with other TLR ligands, specifically with Flagellin, Pam3CSK4, and Poly(I:C) (ligands for TLR5, TLR2, and TLR3, respectively). Similarly to the effect of LPS stimulation, when compared with WT DCs, the $m i R-142^{-/-}$DCs demonstrated reduced expression patterns of MHC class II, CD80, CD86, CD40, and (Figure 1D) pro- and antiinflammatory cytokines (Figure $1 \mathrm{E}$ ), as was noted following LPS stimulation (Figure 1C). 


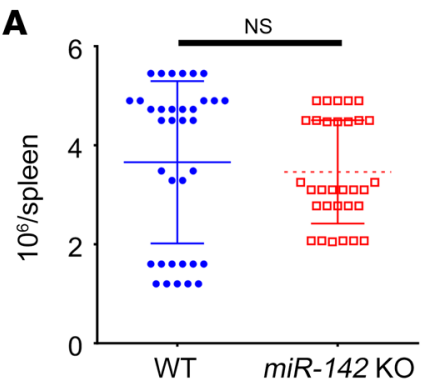

C

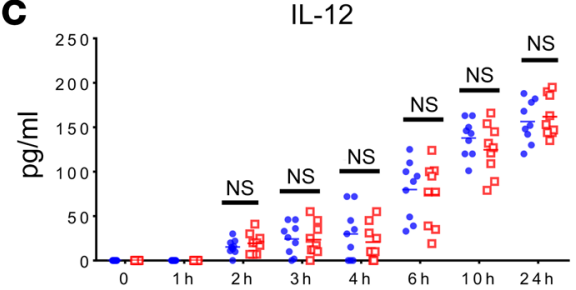

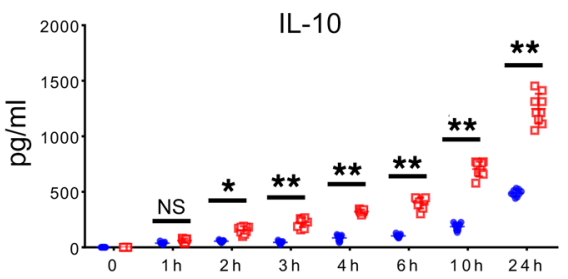
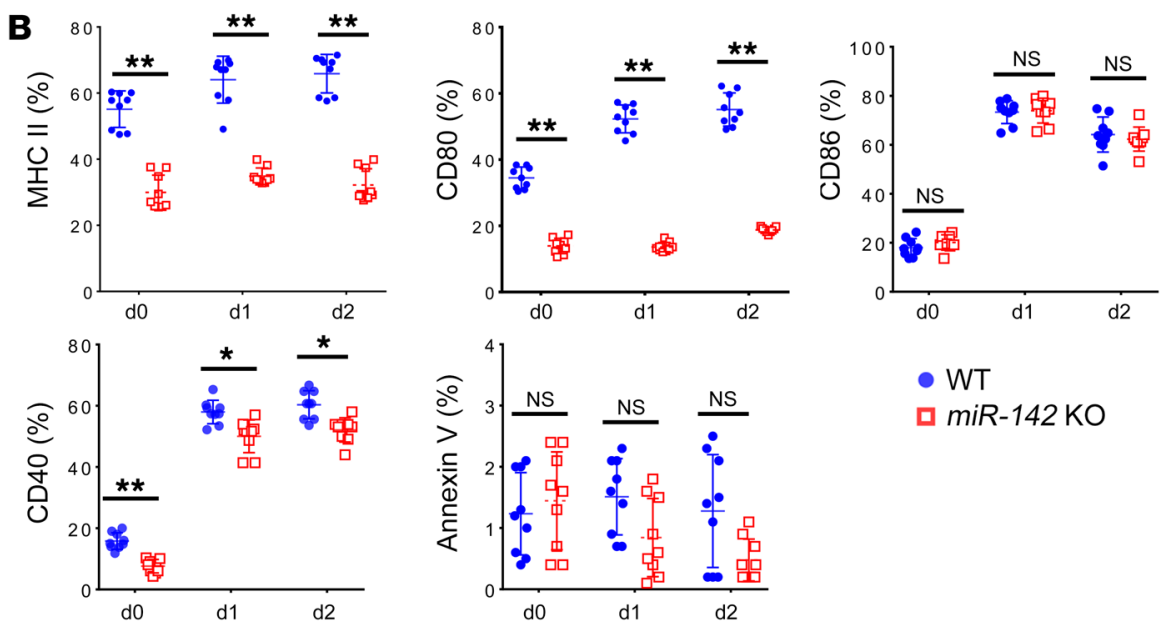

- WT

miR-142 KO
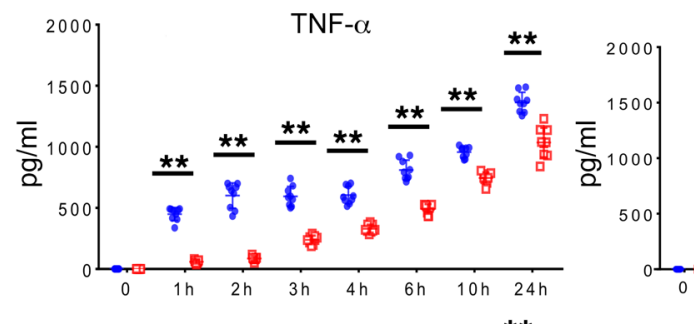

IL-6

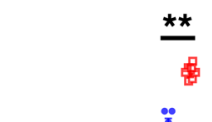
** $\frac{8}{3}$
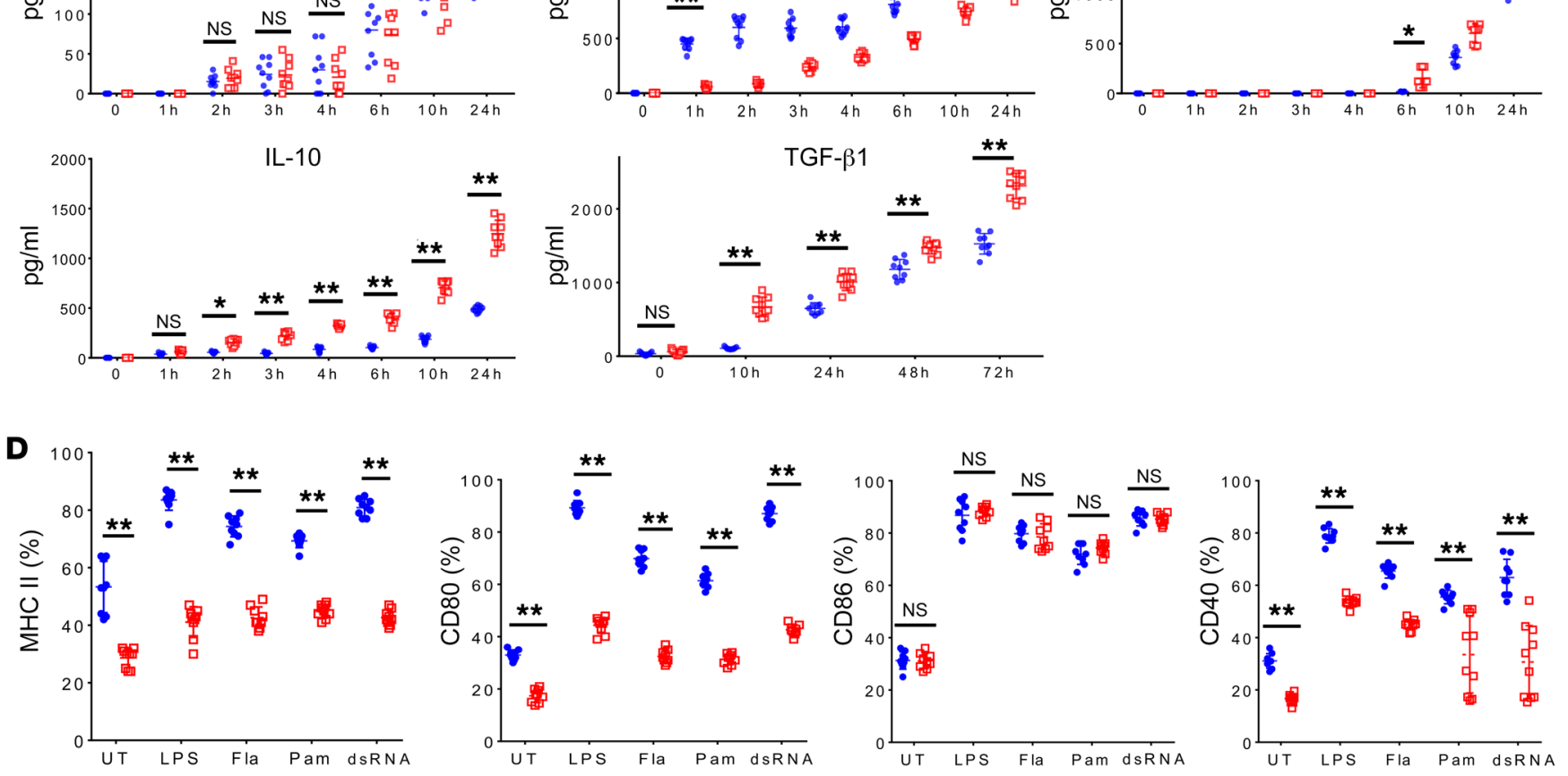

E
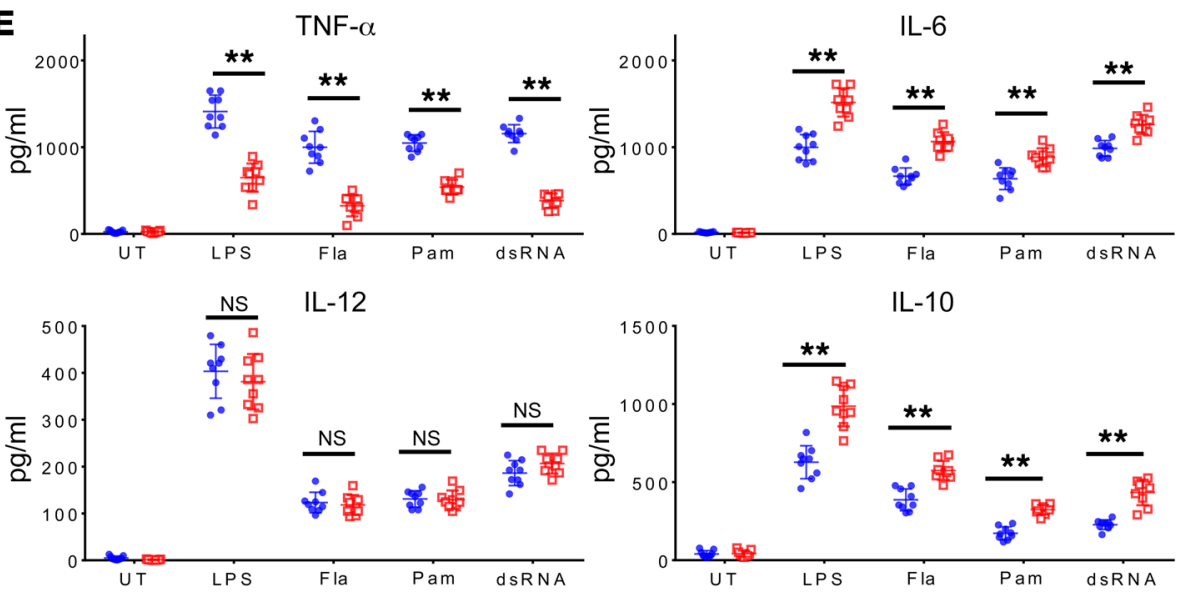

IL-10

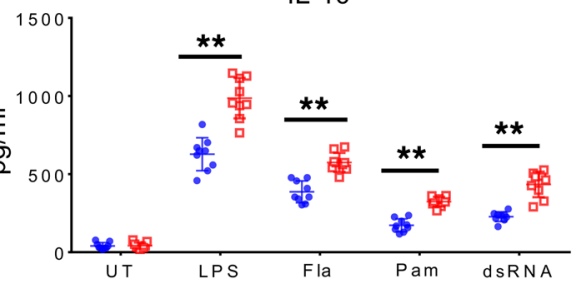


Figure 1. miR-142 modulates DC's TLR responses in vitro. (A) CD11 $\mathrm{c}^{+} \mathrm{DCs}$ were quantified from spleens of WT or miR-142 $2^{-/-}$mice $(n=30$, mean \pm SEM). Splenic WT or miR-142-/- DCs were treated with LPS or diluent control and (B) analyzed for expression of MHC II, CD80, CD86, CD40, annexin $V$ (mean \pm SEM) (data are combined from 4 independent experiments), and (C) cytokine levels by ELISA (mean \pm SEM) (data are combined from 3 independent experiments). (D) Splenic WT or miR-142 ${ }^{-/-}$DCs were treated with TLR ligands LPS, Flagellin, Pam3CSK4, and Poly(I:C) or diluent control and analyzed for expression of MHC II, CD80, CD86, and CD40 (mean \pm SEM) (data are combined from 4 independent experiments) and (E) cytokine levels by ELISA (mean \pm SEM) (data are combined from 3 independent experiments). Comparisons between 2 groups were calculated using paired Student's $t$ test ( 2 tailed), while comparisons between 2 groups at multiple time points were calculated utilizing multiple $t$ tests (Holm-Šidák method). ${ }^{*} P<0.05 ;{ }^{* *} P<0.01$. UT, untreated; Fla, Flagellin; Pam, Pam3CSK4.

The priming of allogeneic (BALB/c) T cells was significantly reduced by $m i R-142^{--}$DCs, as determined by $\mathrm{T}$ cell proliferation (Figure 2A) and secretion of inflammatory cytokines IFN- $\gamma$, IL-2, and IL-17A (Figure 2B), with elevated secretion of IL-10 (Figure 2B). We next determined the in vivo relevance and hypothesized that $m i R-142^{-/}$animals will demonstrate reduction in endotoxin-induced mortality. Consistent with the hypothesis, B 6 miR-142mice demonstrated higher survival rate than B6 WT mice $(60 \%$ versus $20 \%, P<0.03$ ) (Figure 2 C).

Next, we assessed the effect of miR-142 in DCs on in vivo priming of allogeneic T cells. We transferred $3 \times 10^{6} \mathrm{CFSE}$-labeled allogeneic BALB/c T cells into WT and $m i R-142^{-/-}$B 6 mice after lethal total body irradiation (TBI). We observed that proliferation of allogeneic BALB/c T cells was significant lower in $m i R-142^{-/-}$B6 hosts than in the WT B6 host (Figure 3, A-C). To confirm that this reduction in allo-T cells is exclusively due to presence or absence of miR142 only in host DCs (and not confounded by miR-142 absence in other host antigen presenting cells), we next utilized the model in which allogeneic $\mathrm{CD} 4^{+} \mathrm{T}$ cells would respond only to MHC class II allo-antigens on exogenously administered DCs (28). MHC class II-deficient (Abb [H2-Ab1]) B6 mice received 1000 cGy TBI and were then injected with $1 \times 10^{7}$ bone marrow-derived dendritic cells (BMDCs) from syngeneic WT B6 or $m i R-142^{-1-}$ B6 mice. These animals were then transplanted with $2 \times 10^{6} \mathrm{CFSE}$-labeled $\mathrm{CD} 4^{+} \mathrm{T}$ cells from allogeneic bm12 donors, which differ from the recipient $\mathrm{B} 6$ animals by a single MHC class II antigen. Analysis of allogeneic donor $\mathrm{CD}^{+} \mathrm{T}$ cells on day 6 demonstrated significantly lower proliferation and activation in animals that received B6 $\mathrm{miR}$ $142^{-/-}$DCs than in those that received WT DCs (Figure 3, D-G).

We next determined the impact of loss of miR-142 in DCs on generating antigen-specific $\mathrm{T}$ cell responses utilizing OT-I and OT-II systems. Both OT-I- and OT-II-specific T cell responses were reduced when stimulated with $m i R-142^{-/-}$DCs pulsed with $\mathrm{H}-2 \mathrm{~Kb}-$ restricted OVA MHC class I peptide (SIINFEKL) or I-Ad-restricted OVA MHC class II peptide (ISQAVHAAHAEINEAGR), respectively, when compared with similarly pulsed WT DCs (Figure 3H). We next explored whether absence of miR-142 in DCs regulated induction of tolerance in addition to regulation of the priming allogeneic $\mathrm{BALB} / \mathrm{c} \mathrm{T}$ cells. To this end, we primed allogeneic BALB/c T cells in a bulk primary mixed lymphocyte reaction (MLR) with purified B6 WT or B6 miR-142/- DCs. The primed allogeneic BALB/c T cells from the primary cultures for 4 days were then harvested and restimulated with either allogeneic B6 WT or third-party $(\mathrm{C} 3 \mathrm{H} /$ $\mathrm{He}$ ) DCs in a secondary MLR. When compared with the BALB/c $\mathrm{T}$ cells that were primed by the WT B6 DCs, the BALB/c T cells primed with $m i R-142^{-/-}$B6 DCs in the primary MLR demonstrated equivalent proliferation when stimulated by DCs from third-party $(\mathrm{C} 3 \mathrm{H})$, but demonstrated persistent reduced proliferation when restimulated with B6 WT DCs (Figure 3I and Table 1).

Effect of miR-142 on glycolysis in DCs. Recent studies demonstrate that activation of DCs by TLR agonists leads to a rapid upsurge in glycolysis $(6,29)$. We first performed gene-expression studies to analyze the global and, specifically, the metabolic effects of loss of miR-142 in DCs before and after stimulation with LPS. The genes involved in metabolic pathways defined by Ingenuity Pathway Analysis (IPA) (7, 10, 30) were analyzed using Gene Set Enrichment Analysis (GSEA) (15, 17). From a total of 32 genes that were differentially expressed in the glycolysis pathway, 16 were expressed at higher levels and 15 at lower levels at baseline and 9 were upregulated while 14 were downregulated in $\mathrm{miR}-142^{-/-}$ DCs when compared with WT DCs (Figure 4A and Supplemental Figure 2). Moreover, 11 were upregulated and 18 downregulated in LPS-stimulated $m i R-142^{--}$DCs, and 16 were upregulated and 11 downregulated in WT DCs (Figure 4A and Supplemental Figure 2A, and GSE100043) when compared with their baseline.

To determine whether these changes in gene expression have significant functional impact, we measured lactate production, a metabolic end point of aerobic glycolysis, and found an increase in aerobic glycolysis after a 24-hour LPS stimulation that was comparable between WT and $m i R-142^{-/-}$DCs (Figure $4 \mathrm{~B})$. We also observed that both groups had similar sensitivity to inhibition of glycolysis by 2-deoxyglucose (2-DG), an inhibitor of the first enzyme hexokinase (HK) in this pathway (Figure $4 \mathrm{C}$ ). Reverse-transcriptase PCR (RT-PCR) and FACS staining showed comparable upregulation of glucose transporter 1 (Glut1) in both groups following LPS stimulation (Figure 4, D and E). Consistently, we also observed comparable glucose uptake (Figure 4F) and HK2 upregulation in both following LPS treatment (Figure $4 \mathrm{G})$. Furthermore, activation of AMPK was inhibited after LPS stimulation in both WT and $m i R-142^{-/}$DCs (Figure $4 \mathrm{H}$ and Supplemental Figure 2B). Taken together, these data indicate that $m i R-142^{-/-}$DCs retain the ability to undergo glycolysis in response to LPS stimulation. Because AKT/PRAS40/mTOR and STAT3 pathways have been associated with metabolic shifts $(3,31)$, we next explored the impact of miR-142 deficiency on the activation of these pathways. As shown in Figure 4I, both the $m i R-142^{-/-} \mathrm{DCs}$ and WT DCs demonstrated no significant difference in the phosphorylation of AKT/mTOR and STAT3 pathways (Figure 4I and Supplemental Figure 2C).

miR-142 deficiency augments OXPHOS in DCs. While the above data demonstrated the expected glycolytic switch after TLR stimulation in both WT and $m i R-142^{-/-}$DCs, they did not explain the alterations in $m i R-142^{-/-}$DC phenotype and cytokine expression profile at baseline and after LPS treatment. DC activation promotes aerobic glycolysis, but also reduces OXPHOS in DCs $(3-5,8)$. Therefore, to understand the real-time state of glycolysis and the mitochondrial oxygen consumption rate (OCR), we utilized Seahorse XF technology and measured the OCR and extracellular acidification rate (ECAR) ratios (32). 
A

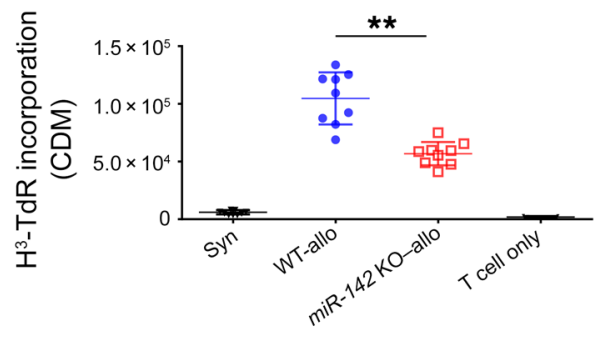

B
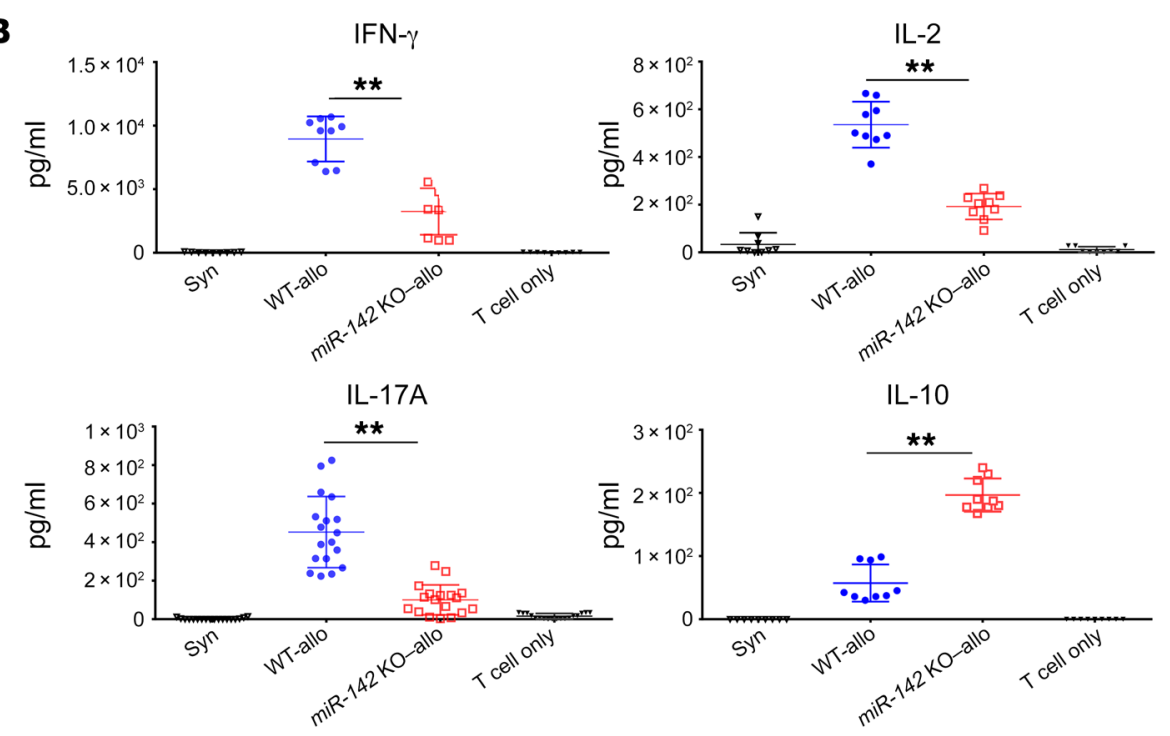

C

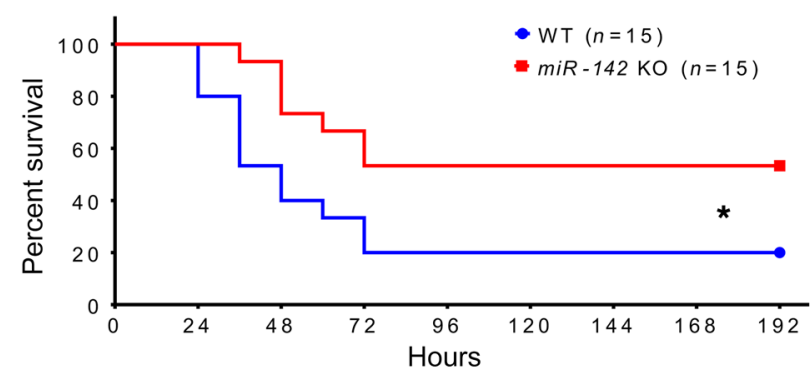

Figure 2. miR-142 modulates DC's priming capability and endotoxin-induced mortality. BALB/c T cells were stimulated by allogeneic B6 WT (WT-allo), miR-142 ${ }^{--}$(miR-142 KO-allo), or syngeneic DCs (Syn) in an MLR reaction. T cell proliferation $(\mathbf{A})$ and cytokine levels $(\mathbf{B})$ were determined (mean \pm SEM) (data shown are combined from 3 similar experiments). (C) LPS was injected i.p. into WT and miR-142-/- mice ( $n=15$ animals/ group), and survival was recorded. Data shown are combined results from 2 independent experiments. Comparisons between 2 groups were calculated using paired Student's $t$ test ( 2 tailed). Survival data were analyzed using log-rank (Mantel-Cox) test. ${ }^{*} P<0.05 ;{ }^{* *} P<0.01$. er levels in $m i R-142^{-/-}$DCs (Figure $4 \mathrm{~B}$ and Supplemental Figure 3) than in WT DCs (Figure 5B and Supplemental Figure 3; Gene Expression Omnibus [GEO] GSE100043). To confirm the results, we measured OCR values in WT DCs, which were significantly reduced after LPS treatment compared with baseline values (Figure $5 \mathrm{C}$ ), consistent with previous reports $(3,4,8)$. Strikingly, in $m i R-142^{-/-} \mathrm{DCs}$, OCR values were significantly higher than WT DCs at steady state (Figure 5C) and increased further after LPS stimulation (Figure 5C). These data suggest that OXPHOS is predominantly enhanced in miR $-142^{-/-}$DCs at baseline and continues to remain higher even after LPS stimulation when compared with that in WT DCs.

Next, to determine whether higher OCR values contribute to higher adenosine triphosphate (ATP) production, we treated DCs with oligomycin to calculate the relative fold change of ATP-linked respiration. In WT DCs, ATP-linked respiration was significantly higher in $m i R-142^{-/-}$DCs than in WT DCs, both at baseline and after LPS stimulation (Figure 5D). We next asked whether the enhanced OXPHOS in $m i R$ $142^{-/-}$DCs is associated with alterations in mitochondrial membrane potential $\left(\Delta \Psi_{M}\right)$ and mitochondrial mass. Both at baseline and after LPS stimulation, the $\Delta \Psi_{\mathrm{M}}$ (Figure 5E) and mitochondrial mass (Figure $5 \mathrm{~F}$ ) were higher in $m i R$ $142^{-/-}$DCs compared with WT DCs. These data suggest that loss of miR-142 in DCs augmented OXPHOS, thus preventing the complete metabolic switch to glycolysis after LPS stimulation.

miR-142 targets CPT1a and regulates $F A O$. Because we found enhanced OCR from augmented OXPHOS (Figure 5) despite comparable levels of glycolysis (Figure 4) and enhanced expression of enzymes that catalyze the TCA cycle in The OCR/ECAR ratio was significantly higher in $m i R-142^{-/-}$DCs treated with LPS than in WT DCs (Figure 5A), indicating robust enhancement of OXPHOS in $m i R-142^{-/-}$DCs.

To better understand the metabolomics underlying the disparate OCR/ECAR ratios between WT and $m i R-142^{-/-} \mathrm{DCs}$, the genes involved in OXPHOS, as defined by IPA and complementary studies $(7,10,30)$, were examined using microarray and GSEA. All levels of genes involved in OXPHOS were significantly higher in $m i R-142^{-/-}$DCs at baseline compared with WT DCs (Figure 5B and Supplemental Figure 3). Following LPS stimulation, these OXPHOS genes continued to be expressed at significantly high-
miR-142 $\%$ DCs (Supplemental Figure 4), we hypothesized that significantly higher OXPHOS in $m i R-142^{-/}$DCs was caused by enhanced FA $\beta$-oxidation (FAO). The genes involved in FAO, as defined by IPA, were analyzed using GSEA. Compared with WT DCs, from a total of 25 genes in the FAO pathway, 18 genes at baseline (Figure 6A and Supplemental Figure 5) and 17 genes after LPS stimulation (Figure 6A and Supplemental Figure 5) were expressed at significantly higher levels in $m i R-142^{-/-}$DCs. Among these, the expression of carnitine palmitoyltransferase -1a (CPT1a), the rate-limiting enzyme of mitochondrial FAO $(33,34)$, was the highest in $m i R-142^{--}$DCs (Figure 6A). Further, computational algo- 

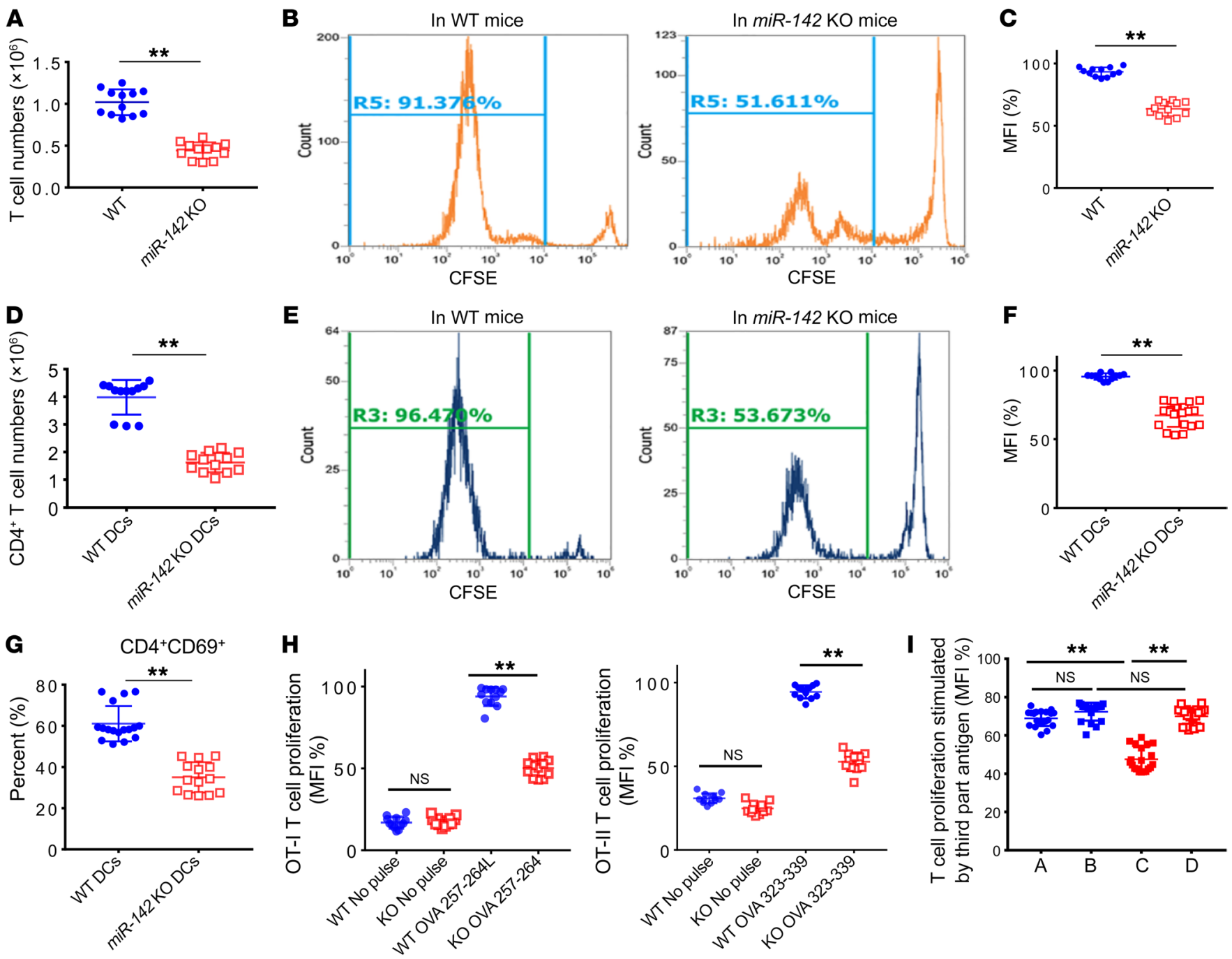

Figure 3. miR-142 modulates DC responses in vivo and ex vivo. (A-C) WT and miR-142-1- B6 mice were irradiated (1000 cGy TBI) on day 0 and injected with 3 $\times 10^{6}$ CFSE-labeled BALB/c T cells on day 1. Three days later, donor T cells were quantified in the recipient spleens and proliferation assessed by CFSE dilution. Data shown are combined from 2 similar experiments (A and C, mean \pm SEM) or a representative of experiments (B). (D-C) Abb (B6) mice were irradiated (1000 cCy TBI) on day 0 and then injected twice with WT and miR-142-/- B6 BMDCs $\left(10 \times 10^{6}\right) 24$ hours apart on days 0 and 1 . Then $2 \times 10^{6}$ CFSE-labeled bm12 CD4+ T cells were transferred on day 1. On day +6 , allogenic $C D 4^{+} T$ cells were analyzed for expansion (D), CFSE dilution analysis (E and $\left.\mathbf{F}\right)$, and T cell activation with $\mathrm{CD}^{+} \mathrm{CD69}^{+}(\mathbf{C})$. Data shown are from combining 2 independent but similar experiments (D, $\mathbf{F}$ and $\mathbf{G}$, mean $\pm \mathrm{SEM}$ ) or a representative of experiments $(\mathbf{E})$. (H) OT-I or OT-II T cell proliferation was assessed by CFSE intensities; data are combined from 2 similar experiments (mean \pm SEM). (I) After first and secondary MLR, BALB/c T cell proliferations were determined by CFSE intensities. Groups were set as indicated in Table 1. Data from 2 similar experiments were combined (mean \pm SEM). Comparisons between 2 groups were calculated using paired Student's $t$ test ( 2 tailed). ${ }^{* *} P<0.01$.

rithms identified CPT1a as putative target for miR-142-3p (Supplemental Figure 6A). Therefore, we next determined whether CPT1a expression is relevant to enhanced FAO in $m i R-142^{-/-}$DCs.

The high expression of CPT1a in miR-142- DCs was confirmed by Western blot and RT-PCR (Figure 6, B and C). Consistent with CPT1a expression, LPS stimulation caused a significant increase in FAO in $m i R-142^{--}$DCs but not in WT DCs (Figure 6D). The knockdown of CPT1a with CPT1a siRNAs that were driven by lentiviruses and a dual convergent promoter in miR-142- - DCs (64\% knockdown efficiency) (Figure 6E) significantly reduced the FAO rate when compared with the cells transduced with a control vector (Figure $6 \mathrm{~F})$. To further determine the direct relevance of CPT1a-dependent FAO on $\mathrm{O}_{2}$ consumption through OXPHOS (35), we measured OCR in WT and KO DCs following treatment with etomoxir, an irrevers-

ible inhibitor of CPT1a (Figure 6G). We found that miR-142- DCs treated with LPS had high FAO weight (Figure 6H), but LPS-stimulated WT DCs showed significantly lower FAO weight (Figure 6H).

\section{Table 1. Group settings for the first and second MLR}

BALB/c T cell preserves responses to third-party antigens

$\begin{array}{llcl}\text { Responders } & \text { First stimulator } & \text { Group } & \begin{array}{l}\text { Second stimulator } \\ \text { BALB/c }\end{array} \\ \text { WT B6 DCs } & \text { A } & \text { WT B6 DCs } \\ \text { T cells } & \text { B } & \text { C3H/He DCs } \\ & \text { miR-142 KO DCs } & \text { C } & \text { WT B6 DCs } \\ & \text { (B6 background) } & \text { D } & \text { C3H/He DCs }\end{array}$


A

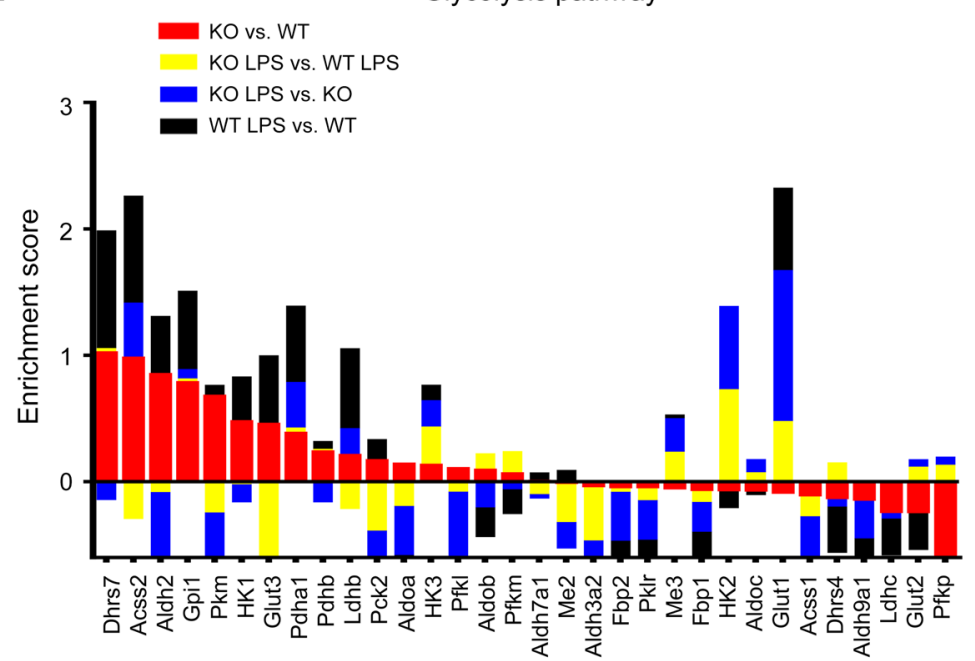

B
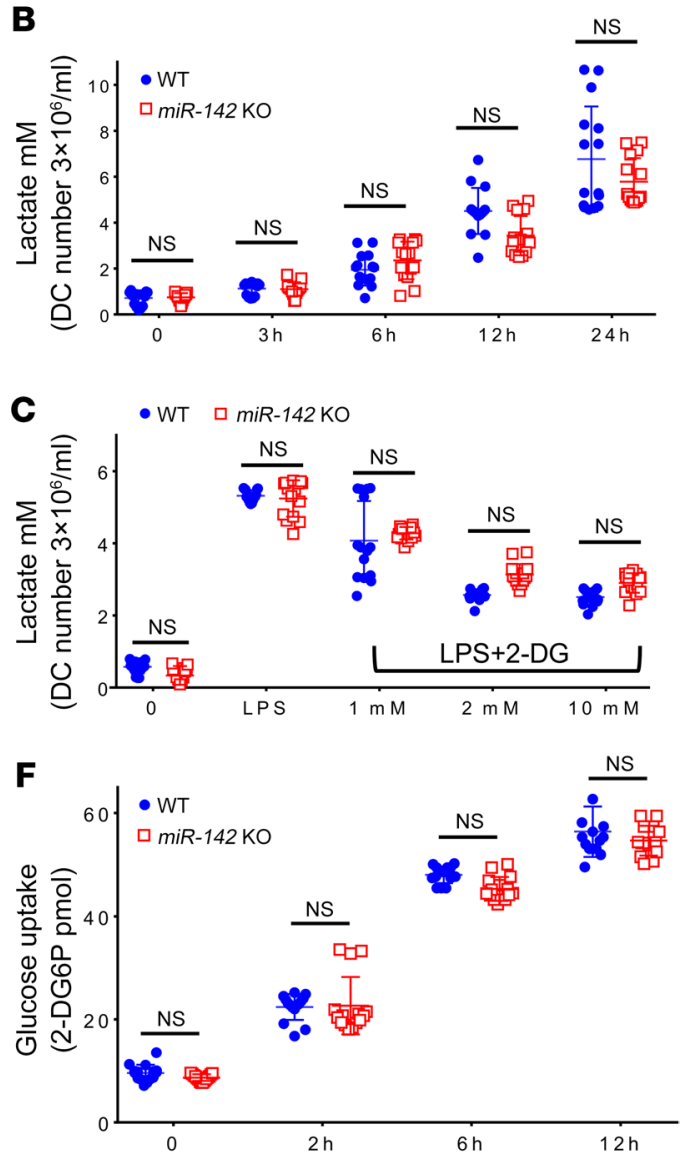

I

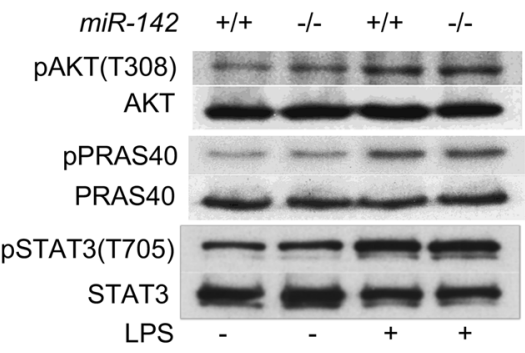

Figure 4. Effect of miR-142 deficiency on glycolysis after LPS stimulation of DCs. (A) Enrichment score of gene set for glycolysis pathway in WT and miR$142^{-/-}$DCs (biological triplicates) by GSEA. (B and C) WT and miR-142-/- DCs were treated with diluent control or LPS and assessed for lactate production without 2-DG (B) and with 2-DG (C). Data (mean \pm SEM) are combined from 3 similar experiments. Expression of CLUT1 in miR-142 $\%$ and WT DCs after LPS treatment was determined for mRNA expression by qPCR (D) and for protein expression by FACS (E) (mean \pm SEM). Data from 3 similar experiments were combined. (F) Glucose uptake (2-DG6P) and (G) HK2 mRNA expression in WT and miR-142-/- DCs treated with LPS. Data (mean \pm SEM) are from 1 of 3 experiments. (H) AMPK activation in WT and $m i R-142^{-/-}$DCs untreated or treated with LPS for indicated times was examined by Western blot. (I) After treatment with LPS, activation of AKT and PRAS (30 minutes) or STAT3 (6 hours) in WT and miR-142-1- DCs was examined by Western blot. Comparisons between 2 groups were calculated using paired Student's $t$ test ( 2 tailed), while comparisons between 2 groups at multiple time points were calculated utilizing multiple $t$ tests (Holm-Šidák method). ${ }^{*} P<0.05$.

These data demonstrate that CPT1a plays a critical role in FAO and that high levels of CPT1a expression in $m i R-142^{-/-}$DCs contribute to sustained high levels of FAO after LPS stimulation.

Regulation of the FA pathway by miR-142 is critical for DC functions. Because the miR-142-- DCs had comparable levels of AMPK activation and glycolysis and glucose uptake, yet demonstrated enhanced OCR (OCR/ECAR), OXPHOS, and FAO rates when compared with WT DCs, we next posited that $m i R-142^{--}$DCs also have the potential for increased FA uptake from the environment as their primary energy source that contributes to alterations in FA metabolism. The miR-142er FA uptake in a time-dependent manner when compared with WT DCs at baseline and after LPS stimulation (Figure 7A and Supplemental Figure 7, A-C). 
A

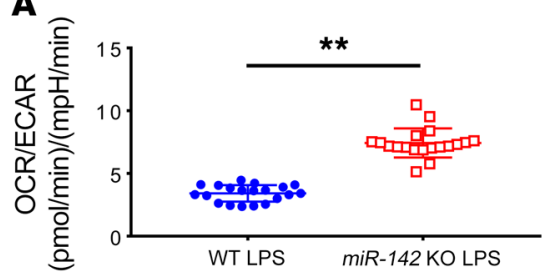

C

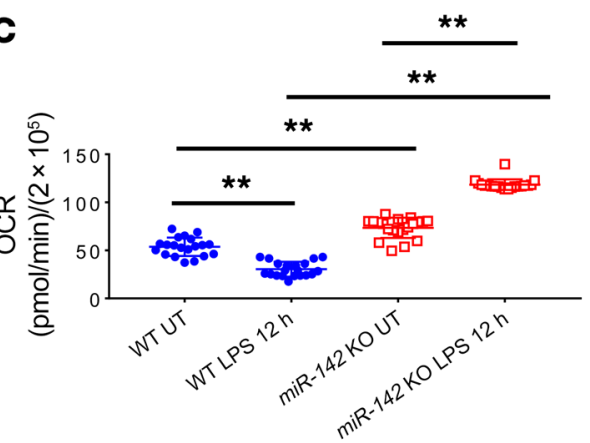

D

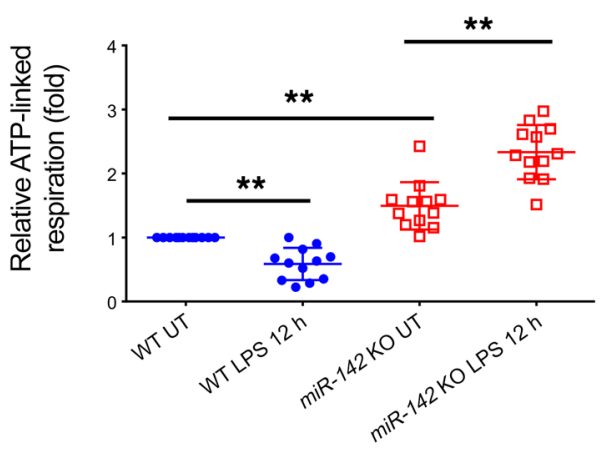

B

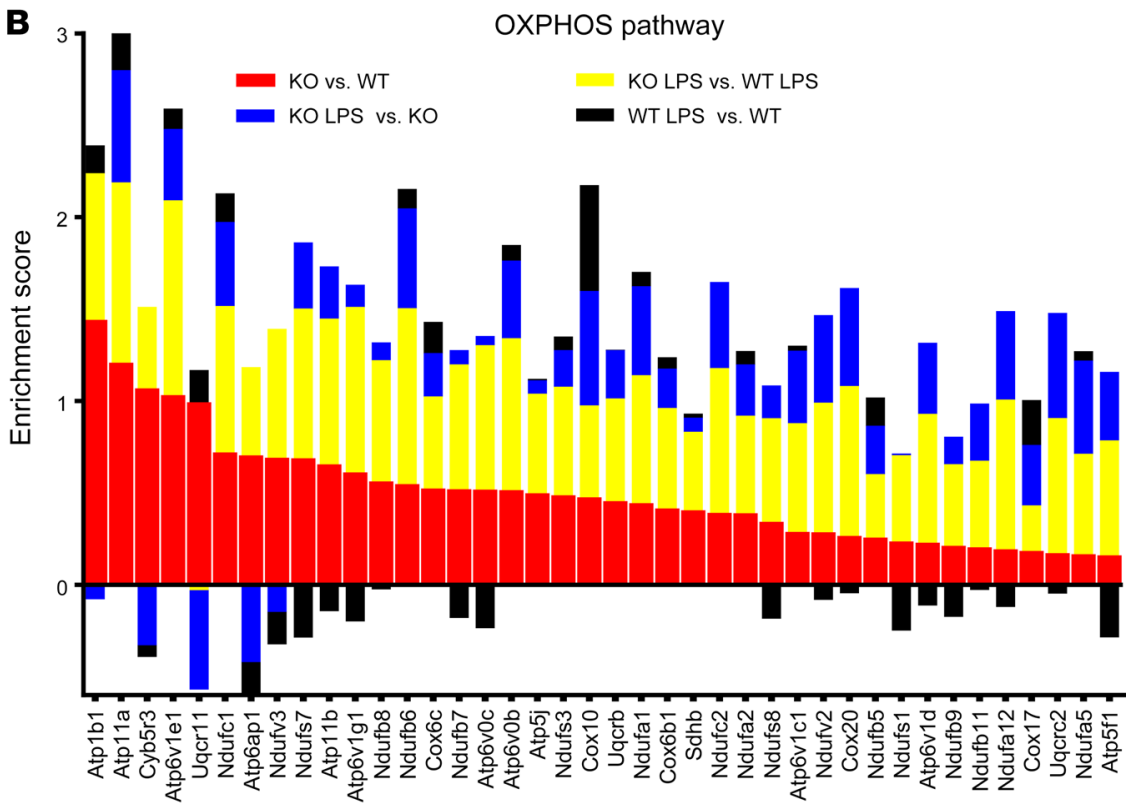

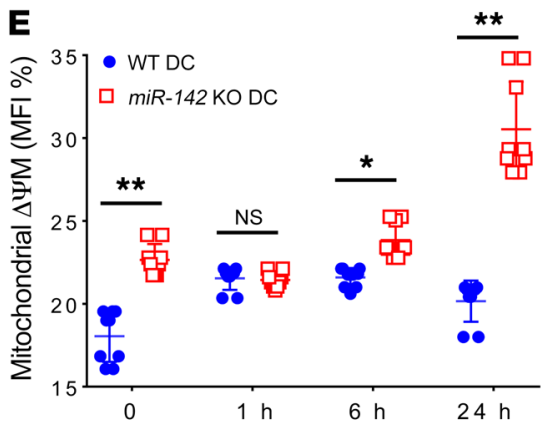

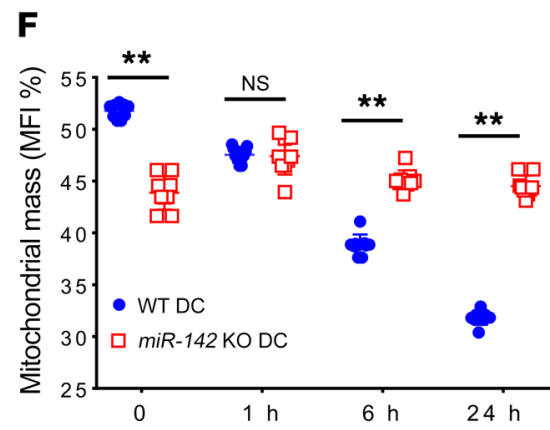

Figure 5. mir-142 regulates OXPHOS in DCs. (A) WT and miR-142-/- DCs were treated with LPS for 6 hours. OCR and ECAR values were recorded by Seahorse, and OCR/ECAR ratios were calculated as described in Methods. Data from 2 similar experiments were combined (mean \pm SEM). (B) Enrichment scores of gene set for OXPHOS pathway in WT and miR-142/- DCs (biological triplicates) by GSEA. (C) WT and miR-142-/- DCs were treated with diluent or LPS for 12 hours. OCR values were pooled from 3 independent experiments (mean \pm SEM). (D) WT and miR-142-- DCs were treated with LPS for 12 hours and then treated with oligomycin. The ATP-linked respiration was calculated as described in Methods. Data from 2 similar experiments were combined (mean \pm SEM). (E and F) WT and miR-142\%- DCs were treated with LPS or diluent for the indicated times and assessed for mitochondrial potential $(\mathbf{E})$ and mass $(\mathbf{F})$ with the MitoTracker. Data from 3 similar experiments were combined (mean \pm SEM). Comparisons between 2 groups were calculated using paired Student's $t$ test (2 tailed), while comparisons between 2 groups at multiple time points were calculated utilizing multiple $t$ tests (Holm-Šidák method). ${ }^{*} P<0.05$; ${ }^{* *} P<0.01$.

We next analyzed the putative causes for enhanced FA uptake (36-40). Microarray studies and GSEA analysis revealed that the FA-binding proteins (FABPs) FABP4 and FABP5 and the FA transporter proteins (FATPs) FATP1 (Slc27a1), FATP4 (Slc27a4), and CD36 were expressed at significantly higher levels in miR$142^{--}$DCs than in WT DCs (Figure 6A; GEO GSE100043). The higher expression levels of these proteins in $m i R-142^{--}$DCs were validated at the protein level (Figure 7, B and C, and Supplemental Figure 8, A and B). Computational algorithms predicted FABP4 and FABP5 to be direct targets of miR-142 (Supplemental Figure 6, B and C). Furthermore, computational algorithms also predicted that Ndufs7 and Uqcrb (components of mitochondrial complexes I and III, respectively) are targets of miR-142 (Supplemental Figure 6, D and E) and found to be expressed at high- er levels in $m i R-142 \%$ DCs than in WT DCs by microarray studies (Figure 4B) and also validated at the protein level (Figure 7D and Supplemental Figure 8C). To confirm the direct and specific impact of these FA uptake proteins as true targets of miR-142 in the regulation of FAO, we first successfully restored miR-142 in $m i R-142^{--}$DCs by transducing miR-142 lentiviral particles (Supplemental Figure 9A). Restoration of miR-142 in miR-142-/DCs reduced the protein expression of CPT1a, FABP4, Ndufs7, and Uqcrb (Figure 7E and Supplemental Figure 9B) and significantly reduced the FAO rate to levels resembling those of WT DCs (Figure 7F). Together, these data suggest that, in addition to direct targeting of CPT1a by miR-142, its direct regulation of these proteins by facilitating FA uptake also contributes to augmented FAO and OXPHOS in $m i R-142^{-/-}$DCs. 


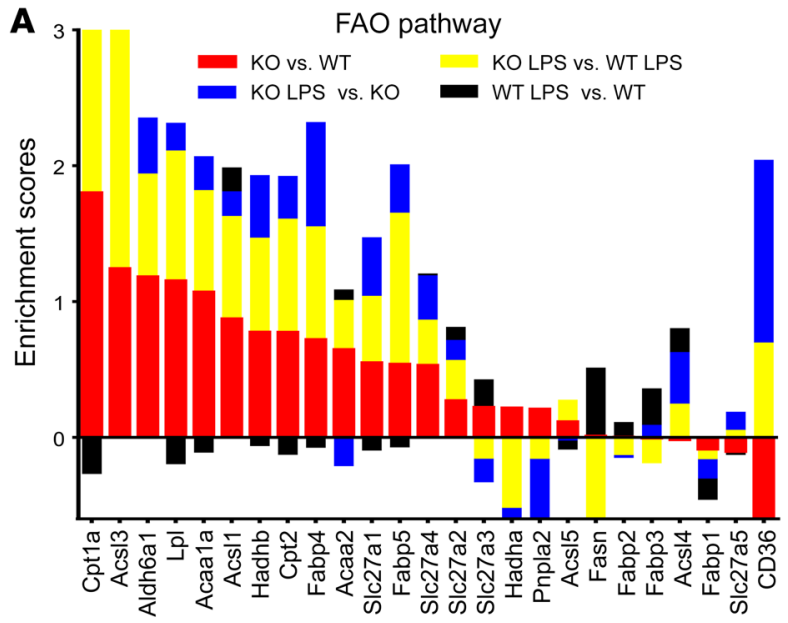

B
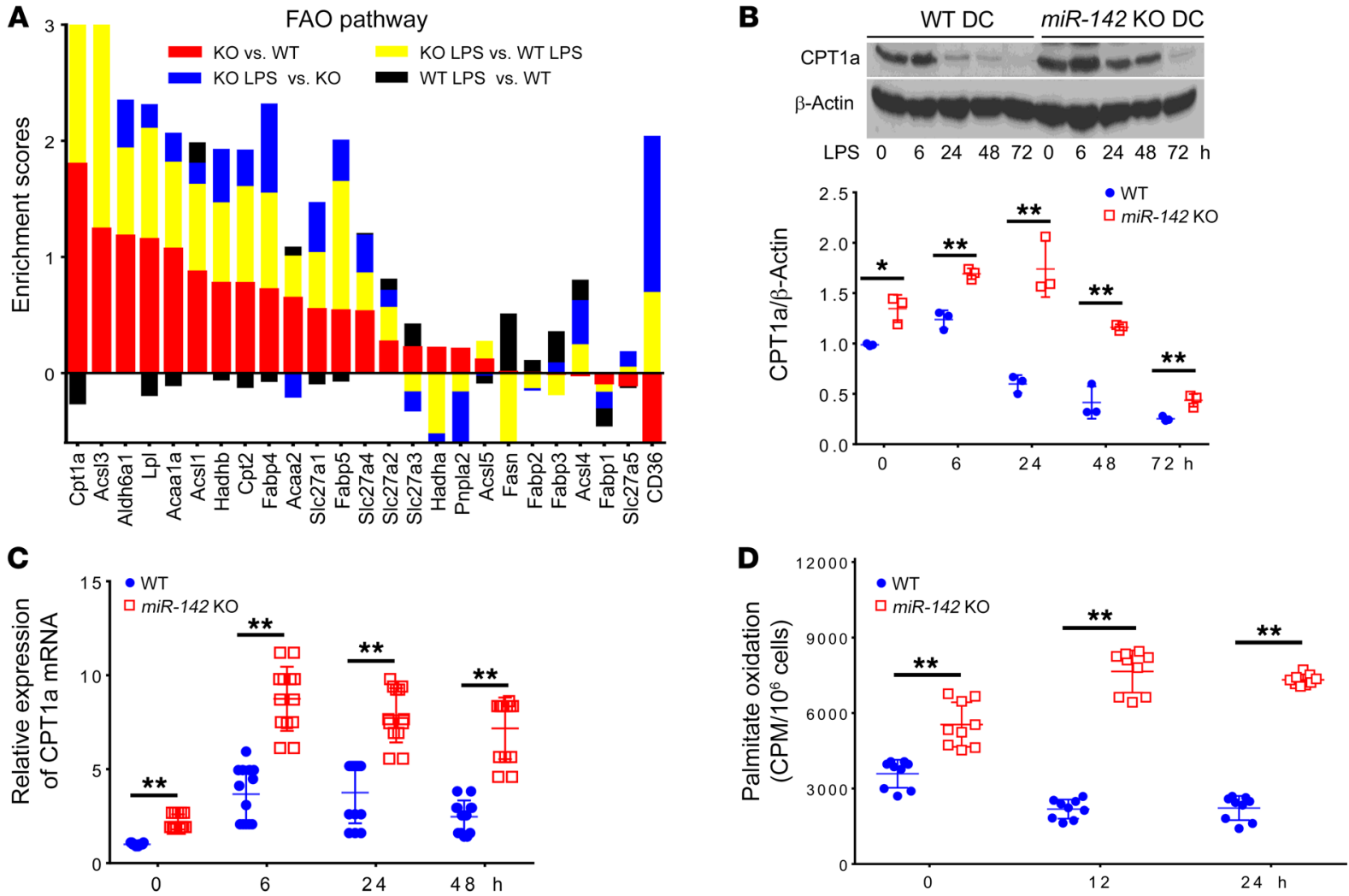

E

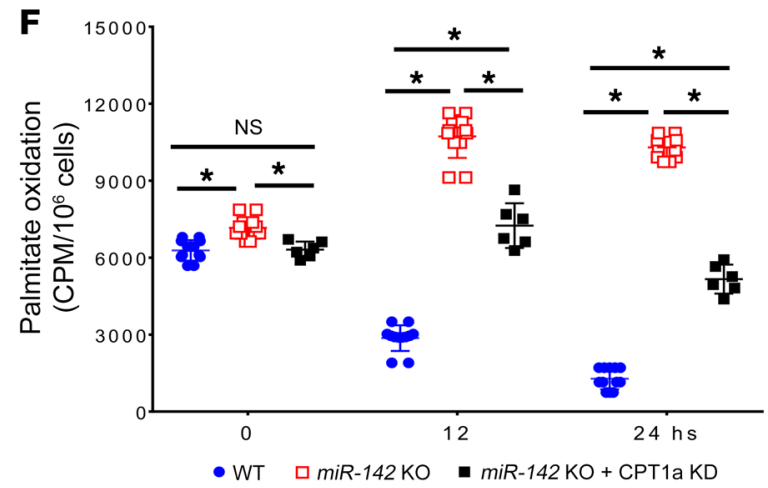

G

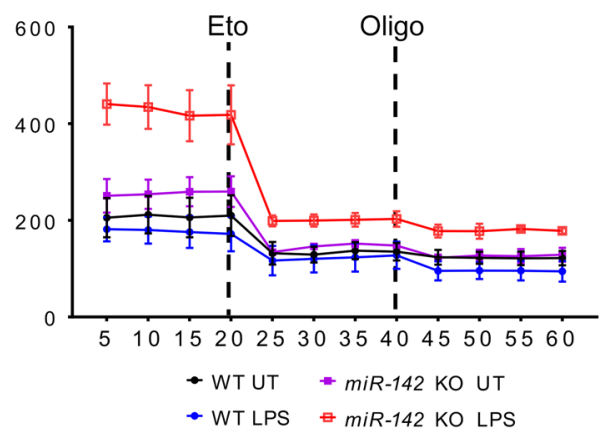

H

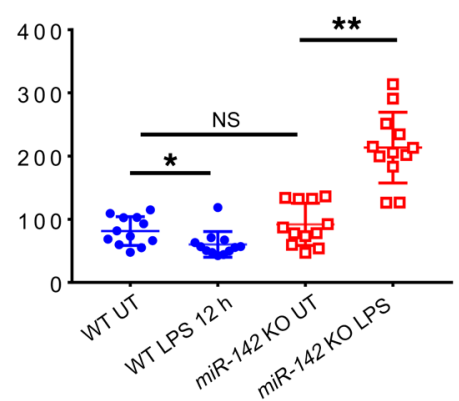


Figure 6. miR-142 targets CPT1a and regulates FAO in DCs. (A) Enrichment score of gene set for FAO pathway in WT and $m i R-142^{-/-}$DCs (biological triplicates) analysis by CSEA. (B) WT and $m i R-142^{---}$DCs were treated with LPS for indicated times. Cell lysates were processed for immunoblotting using Abs against CPT1a and $\beta$-actin. A representative blot is shown (top), and densitometry data (bottom) from 3 independent experiments were combined (mean \pm SEM). (C) WT and miR-142/- DCs treated with LPS for indicated times and processed for qPCR using specific primers for CPT1a. Data from 3 similar experiments were combined (mean \pm SEM). (D) WT and miR-142-/- DCs were treated with LPS for indicated times, then processed for FAO assay as in Methods. CPM values were collected from biological quadruplicate or quintuplet samples. Data from 2 similar experiments were combined (mean \pm SEM). (E) $m i R-142^{-1-}$ DCs were transfected with a pool of CPT1a siRNAs or scramble control as described in Methods. The whole lysates were processed for immunoblotting against CPT1a and $\beta$-actin. A representative blot is shown (top), as are densitometry data combined from 3 independent experiments (mean \pm SEM). (F) WT and miR-142 $1 /$ DCs transfected with lentiviruses, treated with LPS for indicated times, then processed for FAO assay in quadruplicates or quintuplets as described in Methods. Data are combined from 2 independent experiments (mean \pm SEM). (C) WT and miR-142 ${ }^{-1-}$ DCs were treated with LPS for 12 hours, then treated with etomoxir or oligomicin. OCR values were recorded by Seahorse. Data are pooled from 2 independent experiments (mean \pm SEM). (H) OCR values after etomoxir treatment were subtracted from OCR values before etomoxir treatment in WT and miR-142 with or without LPS. Data were pooled from 2 independent experiments (mean \pm SEM). Comparisons between 2 groups were calculated using paired Student's $t$ test ( 2 tailed), while comparisons between 2 groups at multiple time points were calculated utilizing multiple $t$ tests (Holm-Šidák method). ${ }^{*} P<0.05 ;{ }^{* *} P<0.01$.

We next determined whether the miR-142-induced metabolic shifts in OXPHOS and FAO are the cause for the alterations in the functions of $m i R-142^{-/}$DCs (Figures 1 and 2). Using 2-DG, a specific inhibitor for glycolysis, and etomoxir, a specific inhibitor of FAO (41-43), we evaluated the impact on cytokine secretion in WT and $m i R-142^{-/-}$DCs upon LPS stimulation. In both WT and $m i R-142^{-/-}$ DCs, 2-DG significantly reduced the levels of proinflammatory cytokine IL-12, while etomoxir did not produce notable changes in IL-12 expression $(3,4)$ (Figure $7 \mathrm{G}$ and Supplemental Figure 10A). TNF- $\alpha$ expression following LPS stimulation was significantly higher in WT DCs than in miR-142/- DCs, while etomoxir treatment produced comparable levels of TNF- $\alpha$ in both WT and miR-142DCs, indicating that FAO is negatively related to TNF- $\alpha$ induction in $m i R-142^{-/}$DCs (Figure 7G and Supplemental Figure 10B). The expression of IL-10 and TGF- $\beta$ induced by LPS stimulation was significantly inhibited by 2-DG in both WT and $m i R-142^{-/-} \mathrm{DCs}$ (Figure $7 \mathrm{G}$ and Supplemental Figure 10, C and D), demonstrating the dependence on glycolysis (9). However, following treatment with etomoxir, the expression of IL-10 and TGF- $\beta$ in WT DCs was significantly higher than in $m i R-142^{-/-}$DCs (Figure $7 \mathrm{G}$ and Supplemental Figure 10, C and D). Thus, TNF- $\alpha$, IL-10, and TGF- $\beta$ expression are regulated by both glycolysis and FAO in $m i R-142^{-/-}$DCs (39).

\section{Discussion}

Modulation of metabolic pathways has profound effects on DC functions (6). Activation of DCs favors glycolysis over OXPHOS, and this switch is critical for the inflammatory functions of DCs. In contrast, a predominant OXPHOS metabolism has been associated with tolerogenic DCs. The mechanisms that control this metabolic switch and thus the functions of DCs remain poorly understood. miRs have been shown as crucial posttranscriptional regulators of DC functions $(44,45)$, but their role in regulating DC metabolism remains unknown. Herein, we report that miR-142 robustly alters metabolic programming and controls the specificity of DC-mediated immunogenic response.

Upon LPS stimulation, compared with WT DCs, $m i R-142^{-/-}$DCs demonstrated similar glycolysis, but showed a tolerogenic phenotype with reduced expression of surface molecules, such as MHC class II, CD80, CD86, CD40, TNF- $\alpha$, and enhanced expression of IL-10 and TGF- $\beta$. The miR-142/- mice demonstrated reduced inflammation and greater survival following endotoxin-induced sepsis. Furthermore, $m i R-142^{-/-}$DCs demonstrated reduced ability to stimulate allogeneic T cells, both in vitro and in vivo. Seahorse analysis demonstrated that, upon activation of DCs while glycolysis was not impaired, in contrast with WT DCs, the KO DCs continued to depend primarily on OXPHOS. These data demonstrate that deficiency of miR-142 in DCs caused persistence of OXPHOS and are consistent with the notion that a switch away from OXPHOS is required for DC maturation and immunogenicity $(7,10,46)$.

The persistence of OXPHOS in DCs in the absence of miR-142 was associated with augmented FA uptake and FAOs in DCs at baseline and also occurred following activation with LPS. Microarray studies revealed that, while the gene expression profiles for the glycolysis pathway were comparable between WT and $m i R-142^{-1-}$ DCs, the OXPHOS and FAO pathways were enhanced in activated miR-142-/- DCs when compared with those in WT DCs. Computational algorithms predicted that the specific gene, namely CPT1a, that plays a critical role in OXPHOS and FAO and FA uptake, along with other genes such as FABP4, FABP5, Ndufs7, and Uqcrb, act as potential targets of miR-142. Consistent with the predictions, these genes and protein levels were increased in the miR-142-deficient DCs. Restoration of miR-142 in $m i R-142^{-/-}$DCs reduced FAO rates much more than did specific knockdown of CPT1a, indicating that miR-142-mediated metabolic reprogramming is dependent on CPT1a. The rescue experiments of $m i R-142^{-/-D C s}$ with miR-142 reduced not just CPT1a, but also the expression of FABP4, FABP5, Ndufs7, and Uqcrb and attenuated FAO and OXPHOS to the levels observed in WT DCs. Thus, miR-142 acts as the chief regulator of the DC metabolic shift by targeting several key genes in the FA/ OXPHOS pathways. Thus, our data suggest that the net impact of the effect of miR-142 on FAO and OXPHOS is a consequence of its collective effects on other target molecules, such as FABP4, FABP5, Ndufs7, and Uqcrb, in this pathway (Supplemental Figure 6, B-E) in addition to CPT1a (Figure 7E, Supplemental Figure 6A, and Supplemental Figure 10E).

Our data demonstrate that the expression of IL-10 and TGF- $\beta$ is dependent on both glycolysis and FAO in $m i R-142^{--}$DCs. In fact, IL-10 secreted by DCs favors OXPHOS (46). Enhanced FAO in $m i R-142^{-/}$DCs facilitates IL-10 expression, which in turn likely favors OXPHOS, thus forming a positive circuit for IL-10 expression. Our data also show that expression of TNF- $\alpha$ is glycolysis dependent in both WT and $m i R-142^{-/-} \mathrm{DCs}$, indicating a prominent role of glycolysis on TNF- $\alpha$ expression in response to LPS stimulation, while FAO is negatively related to TNF- $\alpha$ induction $(9,47$, 48). Similarly, the increased release of IL-6 might also directly affect FAO metabolism. Although our data were focused exclusively on the impact of direct and cell-intrinsic deficiency of miR- 

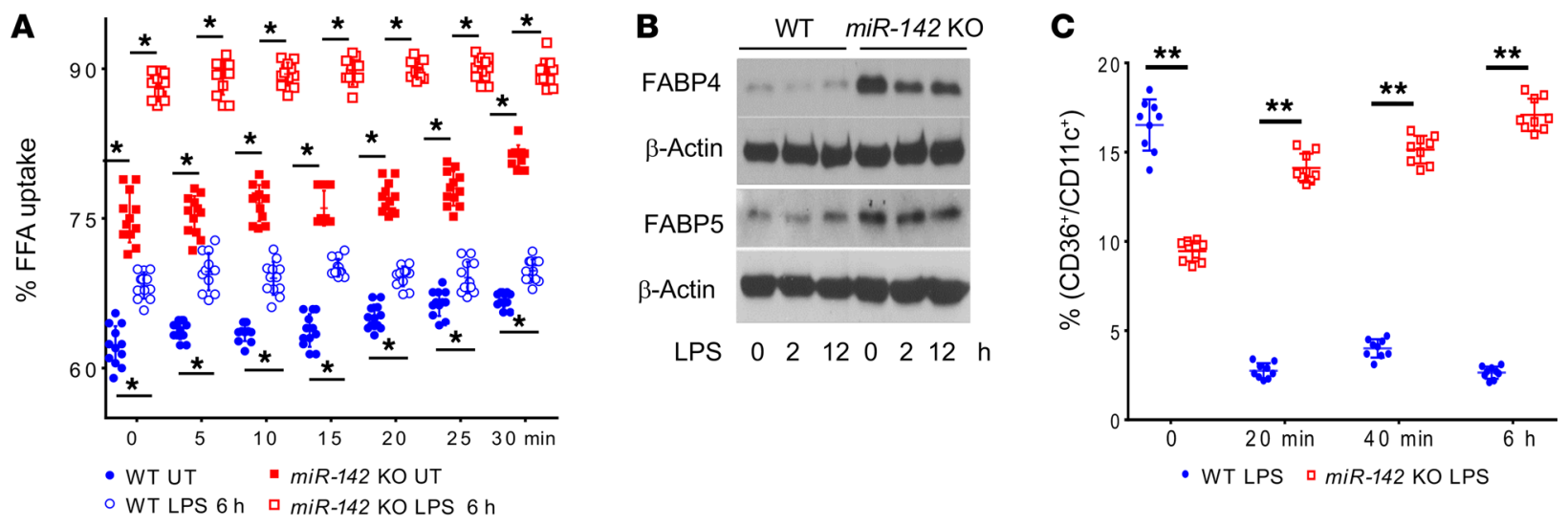

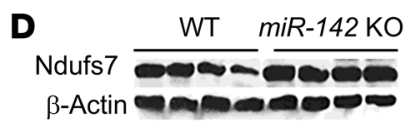

LPS $0 \begin{array}{lllllllll} & 6 & 12 & 0 & 1 & 6 & 12 & \mathrm{~h}\end{array}$
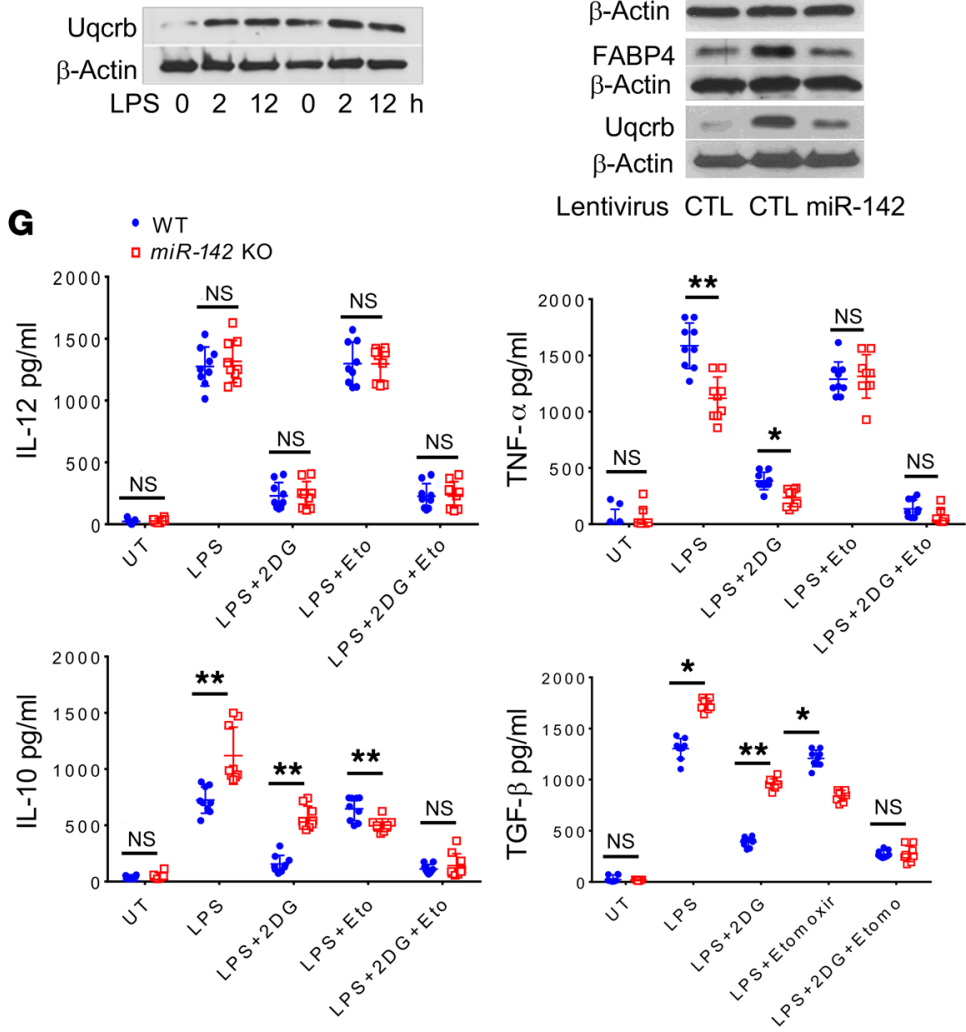

E

Lentivirus CTL CTL miR-142

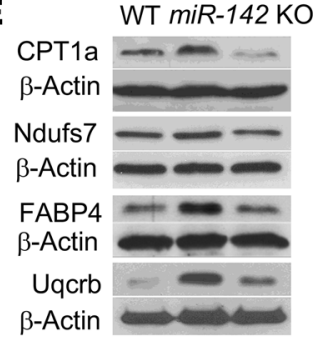

\section{$\mathbf{F}$}

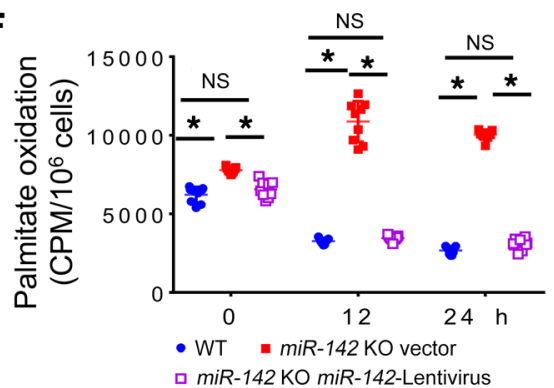

Figure 7. FA uptake is increased in $\mathbf{m i R}-142^{-/-}$DCs. (A) WT and miR-142-/- DCs were treated with diluent control or LPS for 6 hours. FA uptake at 20-second intervals for 30 minutes was determined, as described in Methods. Data from 3 similar experiments were combined (mean \pm SEM). (B-D) WT and miR-142-/DCs were treated with LPS for the indicated times and analyzed by immunoblotting for FABP4, FABP5, $\beta$-actin (B), or by FACS for CD36 expression (C). Data from 3 similar experiments were combined (mean \pm SEM), and immunoblotting was performed using Abs against Ndufs7, Uqcrb, and $\beta$-actin (D). Representative blots are shown. (E) miR-142-/- DCs were transfected with lentiviral miR-142 or control vectors as described in Methods. Cell lysates were processed for immunoblotting using Abs against CPT1a, FABP4, Ndufs7, Uqcrb, and $\beta$-actin. A representative blot is shown. (F) WT and miR-142-/- DCs were transfected with lentiviruses expressing miR-142 or control, treated with LPS for indicated times, then processed for FAO assay. Data from 2 similar experiment were combined (mean $\pm \mathrm{SEM}$ ). Comparisons between 2 groups at multiple time points were calculated utilizing multiple $t$ tests (Holm-Šidák method). ${ }^{*} P<0.05 ;{ }^{*} P<0.01$.
142 in DCs, the complex role of multiple inputs that could regulate metabolism and functions will need to be explored in future studies. Pharmacological manipulation of DC metabolism has shown the potential for clinical application $(7,43,49)$. mTOR inhibition with rapamycin to induce catabolic metabolism favors the generation of tolerogenic DCs (50), and the increase in mitochondrial OXPHOS has been used for the induction of tolerogenic DCs $(3,4$, 51). Enhanced FAO and OXPHOS and tolerogenic characteristics of $m i R-142^{-/-}$DCs suggest that preventing the activity of miR-142 in DCs may have the potential for clinical application in long-term graft acceptance after transplantation and in mitigating graft-versus-host disease (GVHD) or endotoxin sepsis and other immunopathologies characterized by exuberant inflammation.
When we asked whether miR-142 is equally effective in regulating OXPHOS in other cell types beyond DCs, we found that stimulation of $\mathrm{T}$ cells with anti-CD3 and anti-CD28 Abs reduced the expression of CPT1a in WT T cells, while its expression remained constant in $m i R-142^{-/-} \mathrm{T}$ cells (Supplemental Figure 10F) $(32,33)$. Furthermore, stimulation of macrophages with LPS similarly reduced the expression of CPT1a in WT macrophages, but did not alter its expression in $m i R-142^{-/-}$macrophages (Supplemental Figure 10F), which is similar to what occurred in $m i R-142^{-/-}$DCs (Figure $6 \mathrm{~B})$. These data indicate that miR-142 may regulate OXPHOS in other immune cell subtypes beyond DCs. It is important to note that the focus of this study has been exclusively on characterizing the impact on DCs in vitro and in vivo. However, our data are limit- 
ed to analysis of $\mathrm{T}$ cell responses to direct antigen presentation and do not explore the role of metabolic shifts on the complex intracellular antigen-processing pathways of exogenous antigens to $\mathrm{CD} 4^{+}$ $\mathrm{T}$ cells or to $\mathrm{CD} 8^{+} \mathrm{T}$ cells (crosspresentation) or the processing of endogenous antigens to $\mathrm{CD} 8^{+} \mathrm{T}$ cells. Moreover, because the target selection by any given miR might depend on the cellular subset and its context, the exact net effects of miR-142 on the metabolic shifts and bioenergetics of various specific other immune cell subsets, including T cells, Tregs, macrophages, and B cells, will need to be characterized carefully in future studies. Nonetheless, miR142 is emerging as a critical regulator of many biological processes and associated signaling pathways not just in immune cells, but also during embryonic development, homeostasis, and disease. For example, miR-142 deficiency induced defective lymphopoiesis and adaptive immunity (52), ameliorated experimental sepsis and colitis $(14,53)$, and enabled hemodynamic stress-mediated cardiac adaptive hypertrophy (54), while upregulation of miR-142 has been associated with certain cancers $(55,56)$. These studies add to the growing body of work on the critical, but complex, role played by a single microRNA, miR-142, in various biological processes and cell types $(57,58)$.

In summary, our findings show that miR-142 is central to the metabolic reprogramming that specifically favors glycolysis and immunogenic response by DCs.

\section{Methods}

Mice. B6 and BALB/c mice were purchased from Charles River Laboratories. The miR-142-deficient mice (B6 background) were generated by using homologous recombination (15). Abb (B6.129-H2-Ab1tm1Gru N12), Rag2 KO OT-I (model 2234), and OT-II (model 11490) mice were purchased from Taconic. Bm12 (B6.C-H2-Ab1bm12/ $\mathrm{KhEg}-\mathrm{Mc} 1 \mathrm{re}-\mathrm{J} / \mathrm{J})$ and $\mathrm{C} 3 \mathrm{H} / \mathrm{HeJ}\left(\mathrm{H}-2^{\mathrm{k}}\right)$ were purchased from the Jackson Laboratory. The ages of mice ranged between 8 and 12 weeks. Mice were housed in sterilized microisolator cages and given filtered water and normal chow.

Cell-culture media and conditions. For standard cell-culture medium, we used RPMI 1640 medium supplemented with 10\% FBS, penicillin (100 U $3 \mathrm{ml}^{-1}$ ), streptomycin (100 $\mathrm{mg}^{-1} \mathrm{ml}^{-1}$ ), and $55 \mathrm{nM} \mathrm{b2-mer-}$ captoethanol. For low-glucose media, we substituted RPMI 1640 with glucose-free RPMI 1640 (catalog 11879020) and FBS with glucose-free dialyzed FBS (catalog 26400036) from Thermo Fisher Scientific. Cells were cultured at $37^{\circ} \mathrm{C}$ with $5 \% \mathrm{CO}_{2}$, except for cells plated prior to Seahorse assays, which requires incubation without $\mathrm{CO}_{2}$.

$D C$ isolation and generation of BM-derived macrophages. DCs were isolated from splenocytes from B6 miR-142-/- and WT mice and BALB/c mice aged from 10 to 16 weeks. Single-cell suspensions were prepared by repeatedly pipetting smashed spleen tissues, pressing through a cell strainer (Falcon), and then subjecting to mouse CD11c Microbeads (UltraPure; Miltenyi Biotec) for positive selection. The purity of enriched CD $11 c^{+}$DC preparation was 85.6 to approximately $90 \%$. In a few experiments, specifically noted, DCs were generated upon 7-day cultures of BM with GM-CSF and IL-4, as described previously (14). To generate macrophages from BM, BM cells from WT B6 mice or miR-142mice were cultured with murine recombinant GM-CSF (10 ng/ml; BD Biosciences - Pharmingen) and $10 \mathrm{ng} / \mathrm{ml} \mathrm{IL-4} \mathrm{(PeproTech)} \mathrm{for} 7$ to 8 days, harvested, and then positively selected by the CD11 $\mathrm{b}^{+}$microbeads (Miltenyi Biotec).
FACS. DCs treated with or without LPS ( $500 \mathrm{ng} / \mathrm{ml}$, for 1 or 2 days), Flagellin (50 ng/ml for 24 hours), Pam3CSK4 (1 $\mu \mathrm{g} / \mathrm{ml}$ for 24 hours), and Poly(I:C) (50ug/ml for 12 hours) were harvested and suspended in FACS wash buffer (2\% BSA in PBS) and stained with conjugated mAbs (BioLegend), as follows: APC-conjugated $\mathrm{mAb}$ to CD11c, and phycoerythrin-conjugated (PE-conjugated) mAbs to MHC II, CD80, CD86, CD40, annexin V, and CD36. For Glut1 staining, DCs were first incubated with anti-Glut1 mouse mAb (Abcam, catalog ab40084), followed by incubating with secondary Ab-PE goat anti mouse IgG. Experiments were carried out as described previously (3). Briefly, DCs were washed with FACS wash buffer and preincubated with the rat anti-mouse FcR mAb 2.4G2 for 15 minutes at $4^{\circ} \mathrm{C}$ to block nonspecific FcR binding of labeled Abs. Then DCs were incubated with rat anti-mouse FcR mAb (BD Pharmingen, catalog 553142, clone 2.4G2) for 30 minutes at $4^{\circ} \mathrm{C}$. Finally, DCs were washed twice with wash buffer, and analyzed using an Attune NxT Acoustic Cytometer (Thermo Fisher). MFI was calculated from triplicate samples as mean \pm SEM.

ELISA. Concentrations of IL-12, TNF- $\alpha$, IL-10, IL-6, IFN- $\gamma$, IL-2, and IL-17A were measured using ELISA with specific anti-mouse ELISA kits from BD Biosciences, R\&D Systems, or BioLegend as recorded in the Supplemental Table 1. Assays were performed per the manufacturer's protocol and read at $450 \mathrm{~nm}$ in a microplate reader (Bio-Rad). The concentrations were calculated from triplicate samples as mean $\pm \mathrm{SEM}$.

$T$ cell isolation and MLR. T cells were isolated from BALB/c mice by negative selection (Pan T Cell Isolation Kit II; Miltenyi Biotec). T cells were cultured with B6 WT or $m i R-142^{-/-}$DCs or BALB/c DCs at a ratio of 40:1 (T cells versus DCs $3 \times 10^{5}: 7.5 \times 10^{3}$ ) for 96 hours using 96-well flat-bottomed plates (Falcon Labware). Supernatants were collected at 76 hours for measurements of cytokine concentrations by ELISA. Proliferation was determined by incubating the cells with ${ }^{3} \mathrm{H}$-thymidine ( $1 \mathrm{Ci} /$ well $[0.037 \mathrm{MBq}]$ ) for the last 20 hours and counting the cells on a 1205 Betaplate reader (Wallac). OT-I or OT-II T cells were isolated by negative selection and stained with CFSE, cocultured with DCs that were purified from WT B6 or miR-142-/- mice, treated with LPS (500 ng/ml for 3 hours), pulsed with or without H-2Kb-restricted OVA MHC class I epitope (SIINFEKL) or I-Ad-restricted OVA MHC class II epitope (ISQAVHAAHAEINEAGR) $(10 \mu \mathrm{M})$ for 4 hours at a ratio of $1: 8$ for 3 days, then analyzed for $\mathrm{CD} 8^{+}$or $\mathrm{CD} 4^{+} \mathrm{T}$ cell proliferation by CFSE intensity. For third part antigen sensitivity assay, after first MLRs were processed as above, BALB/c T cells were isolated and stained with CFSE and cocultured with DCs purified from WT B6 or $\mathrm{C} 3 \mathrm{H} / \mathrm{HeJ}(\mathrm{H}-2 \mathrm{k})$ mice at a ratio of 30:1 for 4 days. BALB/c proliferation was determined by CFSE intensities.

Endotoxin-induced mortality. WT and miR-142-/- mice were administered with LPS i.p. ( $35 \mathrm{mg} / \mathrm{kg}$ ) in a volume of $700 \mu \mathrm{l}$, and survival was recorded every 3 to 4 hours for the first 72 hours and then daily for 4 to 7 days. Data were analyzed with the log-rank (Mantel-Cox) test.

$T$ cell CFSE labeling, allotransplantation. To examine the priming capability of $m i R-142^{--}$DCs for allogeneic reaction in vivo, BALB/c $\mathrm{T}$ cells were labeled with CFSE at a final concentration of $5 \mu \mathrm{mol} / 1$ according to the manufacturer's instructions (Molecular Probes). After lethal irradiation with $1000 \mathrm{cGy}$ TBI, $3 \times 10^{6} \mathrm{CFSE}-$ labeled BALB/c $\mathrm{T}$ cells were transferred into WT and $m i R-142^{-/-} \mathrm{B} 6$ mice. After 3 days, $\mathrm{T}$ cells were isolated from recipient spleens and $\mathrm{T}$ cell expansion was examined. To specifically investigate the effects of miR-142 on in vivo DC function, a model was devised in which allogeneic $\mathrm{CD} 4^{+} \mathrm{T}$ cells would respond only to MHC class II alloantigens on exogenous- 
ly administered DCs (28). MHC class II-deficient (Abb [H2-Ab1]) B6 mice received $1000 \mathrm{cGy}$ TBI and were injected with $1 \times 10^{7} \mathrm{BMDCs}$ from WT or $m i R-142^{-/-}$mice in 2 doses separated by 24 hours, followed by injection of $2 \times 10^{6}$ CFSE-labeled T cells from allogeneic bm12 donors, which differed from those of the recipient animals by a single MHC class II antigen. At day 6, donor $\mathrm{CD}^{+}{ }^{+} \mathrm{T}$ cells in the spleens were isolated, and cell expansion, proliferation, and activated CD $69^{+} \mathrm{T}$ cells were examined.

Affymetrix microarrays and analyses. tcRNA was extracted from purified WT and $m i R-142^{-/}$DCs with TRIzol LS Reagent (Ambion). After the quality of the total RNA was verified with an Agilent 2100 Bioanalyzer, the samples were processed using the WT-Ovatio Pico System (Affymetrix) and a single round of amplification for samples with even stricter concentration restraints. This system incorporates oligo(dT) and random primers for amplification at the $3^{\prime}$ end throughout the whole transcriptome. Affymetrix mouse genome 4302.0 Arrays (Affymetrix), which contain 45,000 transcripts for annotated genes and expressed sequence tags, were used. The stained arrays were scanned on an Agilent Gene Array Scanner (Affymetrix) with a $560 \mathrm{~nm}$ filter. The data were published and analyzed using the $\mathrm{R}$ statistical environment (http://cran.r-project.org/) provided by Bioconductor (http://www.bioconductor.org/), and quality control was performed by verifying even distribution of PM probes for each chip with no degradation. The expression values were then calculated for each gene using a robust multi-array average (15). This modeling strategy converts the PM probe values into an expression value $\left(\log _{2}\right.$ transformed) for each gene. Metabolic pathways were defined by IPA after initial gene set comparison. The identified gene set involved in a specific metabolic pathway was further analyzed with GSEA (http:// www.broadinstitute.org/gsea/index.jsp). All original microarray data were deposited in the NCBI's GEO database (GSE 100043).

Glycolysis assay. DCs were treated with LPS $(500 \mathrm{ng} / \mathrm{ml})$ for different time points. Media samples were collected and processed for detecting L-lactate with Cayman's glycolysis cell-based assay kit (Cayman Chemical, 600450), according to the manufacturer's instructions, and read at $490 \mathrm{~nm}$ in a microplate reader (Bio-Rad).

Glucose uptake assay. DCs suspended in serum-free medium were treated with LPS $(500 \mathrm{ng} / \mathrm{ml})$ for different time points, then starved in $\mathrm{KRPH} / 2 \%$ BSA for 40 minutes and treated with 2-DG for 20 minutes, followed by cell lysate preparation with extraction buffer and freezing at $-80^{\circ} \mathrm{C}$. The frozen samples were thawed to degrade endogenous NAD (P) and processed for measurement of 2-DG6P at OD $412 \mathrm{~nm}$ in a microplate reader (Bio-Rad) every 2 minutes in kinetic mode according to the manufacturer's instructions (Abcam, ab136955).

PCR. miR and mRNA quantitative real-time PCR (qPCR) were performed as previously described $(14,16,17)$. For miR qPCR, total RNA, including small RNAs, was isolated from DCs using the miRNeasy Mini Kit (QIAGEN). RT was performed by incubating a mixture of $10 \mathrm{ng}$ of RNA, $0.15 \mu \mathrm{l}$ of $100 \mathrm{mM}$ dNTPs, $1 \mu$ l of MultiScribe Reverse Transcriptase (50 U/ $\mu \mathrm{l}), 1.5 \mu \mathrm{l}$ of $10 \times \mathrm{RT}$ Buffer, $0.188 \mu \mathrm{l}$ of RNase Inhibitor $(20 \mathrm{U} / \mu \mathrm{l}), 4.192 \mu \mathrm{l}$ of nuclease-free water, and $3 \mu \mathrm{l}$ of $5 \times$ RT primers specific for miR-142-3p, miR-142-5p and snoRNA135 (internal control) (Applied Biosystems) at $16^{\circ} \mathrm{C}$ for 30 minutes, $42^{\circ} \mathrm{C}$ for 30 minutes, and $85^{\circ} \mathrm{C}$ for 5 minutes. qPCR was performed on an Eppendorf realplex2 system with the following temperature cycles: 1 cycle at $95^{\circ} \mathrm{C}, 10$ minutes, and 40 cycles at $95^{\circ} \mathrm{C}$ for 15 seconds and $60^{\circ} \mathrm{C}$ for 60 seconds. For PCR, each reaction mix contained
$10 \mu \mathrm{l}$ TaqMan 2× Universal PCR Master Mix (no AmpErase UNG), $7.67 \mu \mathrm{l}$ nuclease-free water, $1.33 \mu \mathrm{l}$ RT product, and $1 \mu \mathrm{l} 20 \times$ PCR primers specific for miR-142-3p and snoRNA135 (Thermo Fisher Scientific). All assays were performed in triplicate. PCR amplifications were normalized with internal PCR control snoRNA-135. The threshold level for each experiment was set during the exponential phase of the reaction. For mRNA qPCR, total RNA was isolated from DCs with TRIzol LS Reagent (Ambion). Briefly, $2 \mu \mathrm{g}$ of total RNA was reverse-transcribed into cDNA using the High Capacity cDNA Reverse Transcription Kit (Applied Biosystems) in the presence of random hexamers, SYBR Green Master Mix (Applied Biosystems), and $25 \mathrm{ng}$ of both forward and reverse primers. PCR was performed per the manufacturer's instructions, which were then subjected to melt curve analysis. All reactions were performed in triplicate. The threshold level for each experiment was set during the exponential phase. The DNA in each sample was quantitated by interpolation of its $\mathrm{Ct}$ value from a standard curve of $\mathrm{Ct}$ values. GAPDH was used to normalize the level of gene expression. All oligonucleotide primers are listed in the Supplemental Table 1.

Immunoblot analyses. Lysates were extracted from WT and miR$142^{-/-}$DCs, and 50 to $100 \mu \mathrm{g}$ of protein extract was separated in SDSPAGE and transferred onto a PVDF membrane (GE Healthcare). The membrane was blocked with $5 \%$ nonfat dry milk for 30 minutes and then incubated overnight at $4^{\circ} \mathrm{C}$ with the following Abs in $5 \%$ nonfat milk: anti-AMPK rabbit polyclonal Ab (1:1,000, Cell Signaling, cata$\log 2532)$, anti-pAMPK rabbit polyclonal Ab (1:1,000 in 5\%BSA, Cell Signaling,. catalog 2531), anti-CPT1a rabbit polyclonal Ab (1:1,000, Abcam, catalog ab83862), anti- $\beta$-actin mouse mAb (1:1,000 in 5\% nonfat milk; Abcam, catalog ab8226), anti-FABP4 rabbit polyclonal Ab (1:1,000 in 5\% nonfat milk; Abcam, catalog ab66682), antiFABP5 rabbit polyclonal $\mathrm{Ab}$ (1:1,000 in 5\% nonfat milk, Abcam, catalog ab128650), anti-FATP1 rabbit polyclonal Ab (1:1,000 in 5\% nonfat milk, Abcam, catalog ab81875), anti-FATP4 rabbit polyclonal Ab (1:1,000 in 5\% nonfat milk, Abcam, catalog ab83542), antiNdufs7 rabbit polyclonal Ab (1:500 in 5\% nonfat milk, Proteintech, catalog 15728-1-AP), anti-Uqcrb rabbit polyclonal Ab (1:500 in 5\% nonfat milk, Abcam, catalog ab122827), anti-AKT rabbit polyclonal Ab (1:1000 in 5\% nonfat milk, Cell Signaling Technology, catalog 9272), phosphor-AKT (Thy308) rabbit mAb (1:1,000 in 5\%, BSA, Cell Signaling Technology, catalog 13038), anti-PRAS40 rabbit mAb (1:500 in 5\% nonfat milk, Cell Signaling Technology, catalog 2691), phospho-PRAS40(Thr246) rabbit mAb (1:500 in 5\%, BSA, Cell Signaling Technology, catalog 13175), anti-STAT3 rabbit polyclonal Ab (1:1000 in 5\% nonfat milk, Cell Signaling Technology, catalog 9139) and phospho-STAT3 rabbit mAb (1:1000 in 5\%, BSA, Cell Signaling Technology, catalog 9145). After washing 3 times with TBST for 5 minutes, the blot was incubated with specific HRP-labeled secondary Ab, washed again with TBST, and signals generated and visualized using the Enhanced Chemiluminescence Kit (Thermo Scientific).

ECAR and OCR. DCs were analyzed using an XF-24 Extracellular Flux Analyzer (Seahorse Bioscience). In brief, DCs were plated on XF-24 cell culture plates $\left(5 \times 10^{5}\right.$ cells/well in $\left.200 \mu \mathrm{l}\right)$ and either left unstimulated or stimulated with indicated TLR ligands. At the indicated time points, DCs were washed and analyzed in XF Running Buffer (unbuffered RPMI, 10 mM glucose, $1 \mathrm{mM}$ pyruvate, $5 \% \mathrm{FCS}, 100 \mathrm{U} / \mathrm{ml}$ penicillin/streptomycin, $2 \mathrm{mM}$ L-glutamine), per the manufacturer's instructions, to obtain real-time measurements of OCR and ECAR. 
Where indicated, ECAR and/or OCR was analyzed in response to 1 $\mu \mathrm{M}$ oligomycin, $0.5 \mu \mathrm{M}$ rotenone plus $0.5 \mu \mathrm{M}$ antimycin A (Seahorse Bioscience), and $100 \mu \mathrm{M}$ etomoxir (Sigma-Aldrich). To calculate the ratio of OCR to ECAR, the corresponding OCR and ECAR values were obtained from WT and $m i R-142^{--}$DCs treated with LPS for 12 hours and reported as the mean of the ratios from measurements. To calculate ATP-linked respiration, WT and $m i R-142^{---}$DCs were treated with or without LPS for 12 hours, then treated with oligomycin. First, OCR values after treatment with oligomycin were divided by basal OCR values in WT DCs without LPS treatment; based on this, ATP-linked respiration in rest groups was determined. To calculate FAO weights, OCR values after etomoxir treatment were subtracted from OCR values before etomoxir treatment in WT and $m i R-142^{-}$DCs treated with or without LPS.

Mitochondrial potential and mass. Mitochondrial potential and mass were measured with the use of MitoTracker Red CMXRos (Molecular Probes, M7512) and MitoTracker Green FM (Molecular Probes, M22426), respectively, and flow cytometry was performed according to the manufacturer's instructions.

$\beta$-Oxidation assay. DCs were cultured under the indicated conditions at $1 \times 10^{6}$ cells $/ \mathrm{ml}$. Oxidation of FAs was measured using established methods (33). Briefly, DCs were pelleted and resuspended in medium supplemented with $\left[9,10-{ }^{3} \mathrm{H}\right]$ palmitate (PerkinElmer) complexed with BSA, vortexed for 1 minute, and mixed with a solution containing palmitate and 10\% FA-free BSA (MilliporeSigma, A8806) at a ratio of $1: 2$. A total of $3.3 \mu \mathrm{l}$ of $\left[9,10-{ }^{3} \mathrm{H}\right]$ palmitate $(5 \mathrm{mCi} / \mu \mathrm{l}$ stock) and $6.7 \mu \mathrm{l}$ of BSA were used per $1 \mathrm{ml}$ of cell culture medium, and cells were cultured in 24-well plates with or without etomoxir (200 $\mu \mathrm{M})$. After 24 hours, $0.4 \mathrm{ml}$ supernatant was applied to ion exchange columns (Dowex 1X8-200, MilliporeSigma, 217425), and ${ }^{3} \mathrm{H}_{2} \mathrm{O}$ was eluted with $1 \mathrm{ml}$ (or $2.5 \mathrm{ml}$ ) of $\mathrm{H}_{2} \mathrm{O}$. A $0.4 \mathrm{ml}$ (or $0.75 \mathrm{ml}$ ) aliquot was then mixed with $1 \mathrm{ml}$ scintillation fluid (Ultima Gold, 6013321, PerkinElmer) and counted. Cell number was counted separately from each supernatant, and the results were expressed as counts per minute $(\mathrm{CPM}) / 1 \times 10^{6}$ cells. For each data point, biological quadruplicate or quintuplet samples were set for culture without or with $200 \mu \mathrm{M}$ etomoxir sodium (MilliporeSigma). To calculate the $\beta$-oxidation rate, a ranked pair analysis was used to determine the difference between oxidation counts in the absence and presence of etomoxir. CPM values were recorded on a 1205 Betaplate reader (Wallac).

Lentiviral infection. Lentiviral transduction particles containing CPT1a siRNA or miR-142 or control vector were obtained from Abcam or Vigene. $m i R-142^{-/}$DCs were cultured in complete medium for 24 hours, then replaced with fresh prewarmed complete medium at a concentration of $10^{7}$ cells $/ \mathrm{ml}$ in a final volume of $8 \mathrm{ml}$. DC cells $(2 \mathrm{ml})$ were aliquoted into $15 \mathrm{ml}$ sterile tubes, infected with lentivirus plus polybrene at a concentration of $8 \mu \mathrm{g} / \mathrm{ml}$ with viral transduction enhancer G698 at 1:100, gently mixed, and incubated for 20 minutes at room temperature. After centrifugation, infected DC cells were resuspended in fresh complete medium, plated, and grown for 48 hours. GFP expression was monitored to calculate efficiency of transfection.

FA uptake assay. Uptake of FAs was measured using a free FA uptake assay fluorometric kit (Abcam, ab176768). DCs $\left(1 \times 10^{5}\right.$ \% well), either unstimulated or stimulated with indicated TLR ligands, were resuspended in HBSS and incubated for 30 minutes at $37^{\circ} \mathrm{C}$ in a $\mathrm{CO}_{2}$ incubator, followed by the addition of the fluorescent FA probe. After 1 hour of incubation, fluorescence levels were determined (excitation $485 \mathrm{~nm}$, emission $515 \mathrm{~nm}$ ) using an Attune NxT Acoustic Cytometer (Thermo Fisher).

Statistics. Data were analyzed using GraphPad Prism 7. Comparisons between 2 groups were calculated using paired Student's $t$ test ( 2 tailed), while comparisons between 2 groups at multiple time points were calculated utilizing multiple $t$ tests (the Holm-Šidák method). The log-rank (Mantel-Cox) test was utilized to analyze the survival data. Affymetrix microarray data were analyzed further for specific pathways by IPA. Gene set enrichment for specific gene concepts was ranked by enrichment scores. $P<0.05$ was considered statistically significant.

Study approval. All animal studies were reviewed and approved of by the University Committee on the Use and Care of Animals of the University of Michigan, based on University Laboratory Animal Medicine guidelines.

\section{Author contributions}

YS and PR designed the research, analyzed and interpreted the data, and wrote the manuscript. YS, KO, SB, JW, SK, AT, CZ, HF, and DCP performed the experiments. YS, TS, and PR generated the $m i R-142^{-/-}$mice. RM analyzed the microarray data.

\section{Acknowledgments}

We thank the strong support from research cores at the University of Michigan for transgenic animal model, bioinformatics, DNA sequencing, metabolomics, and laboratory animal medicine. This work was supported by NIH grants HL090775, CA173878, HL128046, and CA203542 (to PR).

Address correspondence to: Pavan Reddy, 7215 CCGC, 1500 E. Medical Center Drive, University of Michigan Rogel Cancer Center, Ann Arbor, Michigan 48109, USA. Phone: 734.936.8785; Email: reddypr@umich.edu.
1. Shortman K, Liu YJ. Mouse and human dendritic cell subtypes. Nat Rev Immunol. 2002;2(3):151-161.

2. Ueno $\mathrm{H}$, et al. Dendritic cell subsets in health and disease. Immunol Rev. 2007;219:118-142.

3. Everts B, et al. TLR-driven early glycolytic reprogramming via the kinases TBK1-IKK $\varepsilon$ supports the anabolic demands of dendritic cell activation. Nat Immunol. 2014;15(4):323-332.

4. Everts B, Pearce EJ. Metabolic control of dendritic cell activation and function: recent advances and clinical implications. Front Immunol. 2014;5:203.
5. Kareva I, Hahnfeldt P. The emerging "hallmarks" of metabolic reprogramming and immune evasion: distinct or linked? Cancer Res. 2013;73(9):2737-2742.

6. Pearce EJ, Everts B. Dendritic cell metabolism. Nat Rev Immunol. 2015;15(1):18-29.

7. Sim WJ, Ahl PJ, Connolly JE. Metabolism is central to tolerogenic dendritic cell function. Mediators Inflamm. 2016;2016:2636701.

8. Everts B, et al. Commitment to glycolysis sustains survival of NO-producing inflammatory dendritic cells. Blood. 2012;120(7):1422-1431.
9. Krawczyk CM, et al. Toll-like receptor-induced changes in glycolytic metabolism regulate dendritic cell activation. Blood. 2010;115(23):4742-4749.

10. Malinarich F, et al. High mitochondrial respiration and glycolytic capacity represent a metabolic phenotype of human tolerogenic dendritic cells. Immunol. 2015;194(11):5174-5186.

11. Baltimore D, Boldin MP, O'Connell RM, Rao DS, Taganov KD. MicroRNAs: new regulators of immune cell development and function. Nat Immunol. 2008;9(8):839-845.

12. Lodish HF, Zhou B, Liu G, Chen CZ. Microman- 
agement of the immune system by microRNAs. Nat Rev Immunol. 2008;8(2):120-130.

13. Xiao C, Rajewsky K. MicroRNA control in the immune system: basic principles. Cell. 2009;136(1):26-36.

14. Sun Y, et al. Targeting of microRNA-142-3p in dendritic cells regulates endotoxin-induced mortality. Blood. 2011;117(23):6172-6183.

15. Sun Y, et al. Mature T cell responses are controlled by microRNA-142. JClin Invest . 2015;125(7):2825-2840.

16. Sun Y, et al. PU.1-dependent transcriptional regulation of miR-142 contributes to its hematopoietic cell-specific expression and modulation of IL-6. JImmunol. 2013;190(8):4005-4013.

17. Sun $\mathrm{Y}$, et al. Allogeneic $\mathrm{T}$ cell responses are regulated by a specific miRNA-mRNA network. JClin Invest. 2013;123(11):4739-4754.

18. Sonda N, et al. miR-142-3p prevents macrophage differentiation during cancer-induced myelopoiesis. Immunity. 2013;38(6):1236-1249.

19. Mildner A, et al. Mononuclear phagocyte miRNome analysis identifies miR-142 as critical regulator of murine dendritic cell homeostasis. Blood. 2013;121(6):1016-1027.

20. Beltrán-Anaya FO, Cedro-Tanda A, HidalgoMiranda A, Romero-Cordoba SL. Insights into the regulatory role of non-coding RNAs in cancer metabolism. Front Physiol. 2016;7:342.

21. Alrob OA, Khatib S, Naser SA. MicroRNAs 33, 122, and 208: a potential novel targets in the treatment of obesity, diabetes, and heart-related diseases. J Physiol Biochem. 2017;73(2):307-314.

22. Desgagné V, Bouchard L, Guérin R. microRNAs in lipoprotein and lipid metabolism: from biological function to clinical application. Clin Chem Lab Med. 2017;55(5):667-686.

23. Rottiers V, Näär AM. MicroRNAs in metabolism and metabolic disorders. Nat Rev Mol Cell Biol. 2012;13(4):239-250.

24. Trajkovski M, et al. MicroRNAs 103 and 107 regulate insulin sensitivity. Nature. 2011;474(7353):649-653.

25. Xie H, Lim B, Lodish HF. MicroRNAs induced during adipogenesis that accelerate fat cell development are downregulated in obesity. Diabetes. 2009;58(5):1050-1057.

26. Najafi-Shoushtari SH, et al. MicroRNA-33 and the SREBP host genes cooperate to control cholesterol homeostasis. Science. 2010;328(5985):1566-1569.

27. Ding J, et al. Effect of miR-34a in regulating steatosis by targeting PPAR $\alpha$ expression in nonalcoholic fatty liver disease. Sci Rep. 2015;5:13729.

28. Mathewson N, et al. Neddylation plays an important role in the regulation of murine and human dendritic cell function. Blood. 2013;122(12):2062-2073.
29. Vanherwegen AS, Gysemans C, Overbergh L. Dendritic cell metabolism: immunity and tolerance. Oncotarget. 2015;6(33):34039-34040.

30. Schinnerling K, García-González P, Aguillón JC. Gene expression profiling of human monocyte-derived dendritic cells - searching for molecular regulators of tolerogenicity. Front Immunol. 2015;6:528.

31. Poli V, Camporeale A. STAT3-mediated metabolic reprograming in cellular transformation and implications for drug resistance. Front Oncol. 2015;5:121.

32. Zhang J, et al. Measuring energy metabolism in cultured cells, including human pluripotent stem cells and differentiated cells. Nat Protoc. 2012;7(6):1068-1085.

33. Patsoukis N, et al. PD-1 alters T-cell metabolic reprogramming by inhibiting glycolysis and promoting lipolysis and fatty acid oxidation. Nat Commun. 2015;6:6692.

34. van der Windt GJ, et al. Mitochondrial respiratory capacity is a critical regulator of CD8+ T cell memory development. Immunity. 2012;36(1):68-78.

35. Grynberg A, Demaison L. Fatty acid oxidation in the heart. JCardiovasc Pharmacol. 1996; 28 Suppl 1:S11-S17.

36. Glatz JF. Lipids and lipid binding proteins: a perfect match. Prostaglandins Leukot Essent Fatty Acids. 2015;93:45-49.

37. Glatz JF, Luiken JJ, Bonen A. Membrane fatty acid transporters as regulators of lipid metabolism: implications for metabolic disease. Physiol Rev. 2010;90(1):367-417.

38. Harasim E, Kalinowska A, Chabowski A, Stepek T. [The role of fatty-acid transport proteins (FAT/ CD36, FABPpm, FATP) in lipid metabolism in skeletal muscles]. Postepy Hig Med Dosw (Online). 2008;62:433-441.

39. Hossain F, et al. Inhibition of fatty acid oxidation modulates immunosuppressive functions of myeloid-derived suppressor cells and enhances cancer therapies. Cancer Immunol Res. 2015;3(11):1236-1247.

40. Hotamisligil GS, Bernlohr DA. Metabolic functions of FABPs--mechanisms and therapeutic implications. Nat Rev Endocrinol. 2015;11(10):592-605.

41. Griffin MD, Lutz W, Phan VA, Bachman LA, McKean DJ, Kumar R. Dendritic cell modulation by 1alpha, 25 dihydroxyvitamin D3 and its analogs: a vitamin D receptor-dependent pathway that promotes a persistent state of immaturity in vitro and in vivo. Proc Natl Acad Sci U S A. 2001;98(12):6800-6805.

42. Maldonado RA, von Andrian UH. How tolerogenic dendritic cells induce regulatory $\mathrm{T}$ cells. $A d v$ Immunol. 2010;108:111-165.
43. Morelli AE, Thomson AW. Tolerogenic dendritic cells and the quest for transplant tolerance. Nat Rev Immunol. 2007;7(8):610-621.

44. Mehta A, Baltimore D. MicroRNAs as regulatory elements in immune system logic. Nat Rev Immunol. 2016;16(5):279-294.

45. Zhou H, Wu L. The development and function of dendritic cell populations and their regulation by miRNAs. Protein Cell. 2017;8(7):501-513.

46. Ibrahim J, et al. Dendritic cell populations with different concentrations of lipid regulate tolerance and immunity in mouse and human liver. Gastroenterology. 2012;143(4):1061-1072.

47. Gao X, et al. Carnitine palmitoyltransferase $1 \mathrm{~A}$ prevents fatty acid-induced adipocyte dysfunction through suppression of c-Jun $\mathrm{N}$-terminal kinase. Biochem J. 2011;435(3):723-732.

48. Malandrino MI, et al. Enhanced fatty acid oxidation in adipocytes and macrophages reduces lipid-induced triglyceride accumulation and inflammation. Am J Physiol Endocrinol Metab. 2015;308(9):E756-E769.

49. Nikolic T, Roep BO. Regulatory multitasking of tolerogenic dendritic cells - lessons taken from vitamin $\mathrm{d} 3$-treated tolerogenic dendritic cells. Front Immunol. 2013;4:113.

50. Majumder PK, et al. mTOR inhibition reverses Akt-dependent prostate intraepithelial neoplasia through regulation of apoptotic and HIF-1-dependent pathways. Nat Med. 2004;10(6):594-601.

51. Lagouge $\mathrm{M}$, et al. Resveratrol improves mitochondrial function and protects against metabolic disease by activating SIRT1 and PGC-1alpha. Cell. 2006;127(6):1109-1122.

52. Kramer NJ, et al. Altered lymphopoiesis and immunodeficiency in miR-142 null mice. Blood. 2015;125(24):3720-3730.

53. Duijvis NW, et al. Inhibition of miR-142-5P ameliorates disease in mouse models of experimental colitis. PLOS ONE. 2017;12(10):e0185097.

54. Sharma S, Liu J, Wei J, Yuan H, Zhang T, Bishopric NH. Repression of miR-142 by $\mathrm{p} 300$ and MAPK is required for survival signalling via gp130 during adaptive hypertrophy. EMBO Mol Med. 2012;4(7):617-632.

55. Kaduthanam S, et al. Serum miR-142-3p is associated with early relapse in operable lung adenocarcinoma patients. Lung Cancer. 2013;80(2):223-227.

56. Isobe $\mathrm{T}$, et al. miR-142 regulates the tumorigenicity of human breast cancer stem cells through the canonical WNT signaling pathway. Elife. 2014;3:e01977.

57. Sharma S. Immunomodulation: a definitive role of microRNA-142. Dev Comp Immunol. 2017;77:150-156.

58. Shrestha A, et al. MicroRNA-142 is a multifaceted regulator in organogenesis, homeostasis, and disease. Dev Dyn. 2017;246(4):285-290. 